\title{
LysM Receptor-Like Kinase and LysM Receptor-Like Protein Families: An Update on Phylogeny and Functional Characterization
}

\author{
Luis Buendia, Ariane Girardin, Tongming Wang, Ludovic Cottret and Benoit Lefebvre* \\ Laboratoire des Interactions Plantes-Microorganismes, INRA, CNRS, Université de Toulouse, Castanet-Tolosan, France
}

OPEN ACCESS

Edited by:

Adi Avni,

Tel Aviv University, Israel

Reviewed by:

Erik Limpens,

Wageningen University \& Research,

Netherlands

Yusuke Saijo,

Nara Institute of Science and Technology (NAIST), Japan

${ }^{*}$ Correspondence:

Benoit Lefebvre

benoit.lefebvre@inra.fr

Specialty section:

This article was submitted to

Plant Microbe Interactions,

a section of the journal

Frontiers in Plant Science

Received: 22 June 2018

Accepted: 28 September 2018

Published: 24 October 2018

Citation:

Buendia L, Girardin A, Wang T,

Cottret $L$ and Lefebvre $B$ (2018) LysM

Receptor-Like Kinase and LysM

Receptor-Like Protein Families: An

Update on Phylogeny and Functional

Characterization.

Front. Plant Sci. 9:1531.

doi: 10.3389/fpls.2018.01531
Members of plant specific families of receptor-like kinases (RLKs) and receptor-like proteins (RLPs), containing 3 extracellular LysMs have been shown to directly bind and/or to be involved in perception of lipo-chitooligosaccharides (LCO), chitooligosaccharides (CO), and peptidoglycan (PGN), three types of GlcNAc-containing molecules produced by microorganisms. These receptors are involved in microorganism perception by plants and can activate different plant responses leading either to symbiosis establishment or to defense responses against pathogens. LysM-RLK/Ps belong to multigenic families. Here, we provide a phylogeny of these families in eight plant species, including dicotyledons and monocotyledons, and we discuss known or putative biological roles of the members in each of the identified phylogenetic groups. We also report and discuss known biochemical properties of the LysM-RLK/Ps.

Keywords: defense, symbiosis, MAMP, symbiotic signal, receptor ligand interaction

\section{INTRODUCTION}

\section{Plant Receptor-Like Kinases and Receptor-Like Proteins}

Receptor-like kinases (RLKs) are PM proteins found in most eukaryotic organisms. They are transmembrane proteins with an ECR containing a sensor domain, a TM and an ICR containing a domain with homology to protein kinases, involved in signal transduction (Figure 1A). RLKs sense the extracellular environment. They are found in animals, but their number is particularly high in plants in which they have been mainly described to be involved in perception of beneficial or pathogenic microbes (for review, Antolín-Llovera et al., 2012) and in cell/organ communication (for review, Belkhadir et al., 2014; Hazak and Hardtke, 2016). Several RLKs have also been shown to play a role during abiotic stress (for review, Ye et al., 2017). Plant RLKs are divided in subfamilies depending on their ECRs (Shiu and Bleecker, 2003). Among these families, one bears three LysM on the ECR. This subfamily is the main subject of this review.

\footnotetext{
Abbreviations: AA, amino acids; AMF, arbuscular mycorrhizal fungi; AMS, arbuscular mycorrhizal symbiosis; CO, chitooligosaccharide; CSSP, common symbiosis signaling pathway; ECR, extracellular region; EPS, exopolysaccharide; ER, endoplasmic reticulum; ETI, effector-triggered immunity; GlcNAc, $N$-acetyl glucosamine; GPI, glycosylphosphatidylinositol; ICR, intracellular region; IT, infection thread; ITC, isothermal titration calorimetry; LCO, lipo-chitooligosaccharide; LPS, lipopolysaccharide; LRR, leucine rich repeat; LYK, LysM domain-containing receptor-like kinases; LYM, LysM-proteins; LYR, LYK-related; LysM, lysin motif; MAMP, microbe-associated molecular pattern; MST, microscale thermophoresis; MTI, MAMP-triggered immunity; NMR, nuclear magnetic resonance; PGN, peptidoglycan; PM, plasma membrane; RLK, receptor-like kinase; RLP, receptor-like protein; RNAi, RNA interference; RNS, root nodule symbiosis; ROS, reactive oxygen species; SP, signal peptide; TM, transmembrane domain; VIGS, virus induced gene silencing; WT, wild type.
} 


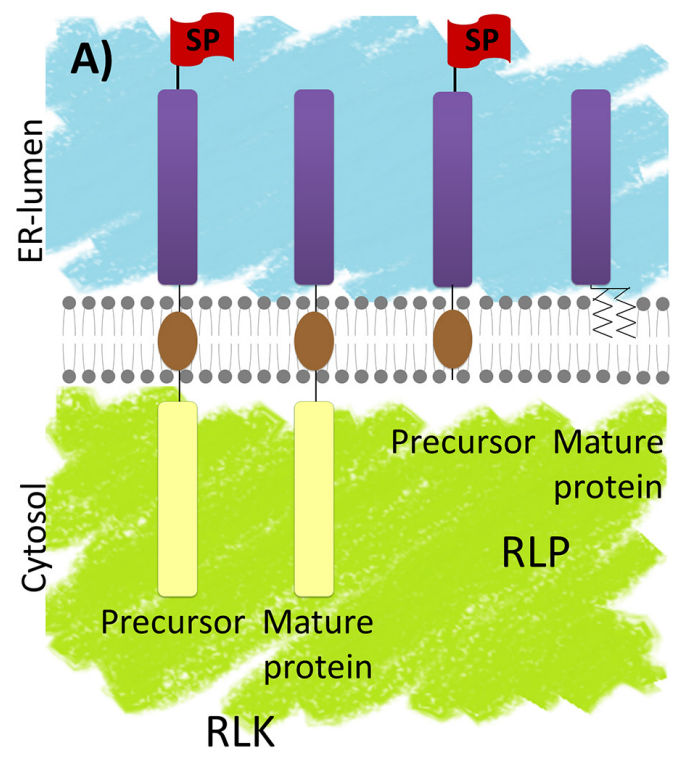

C)

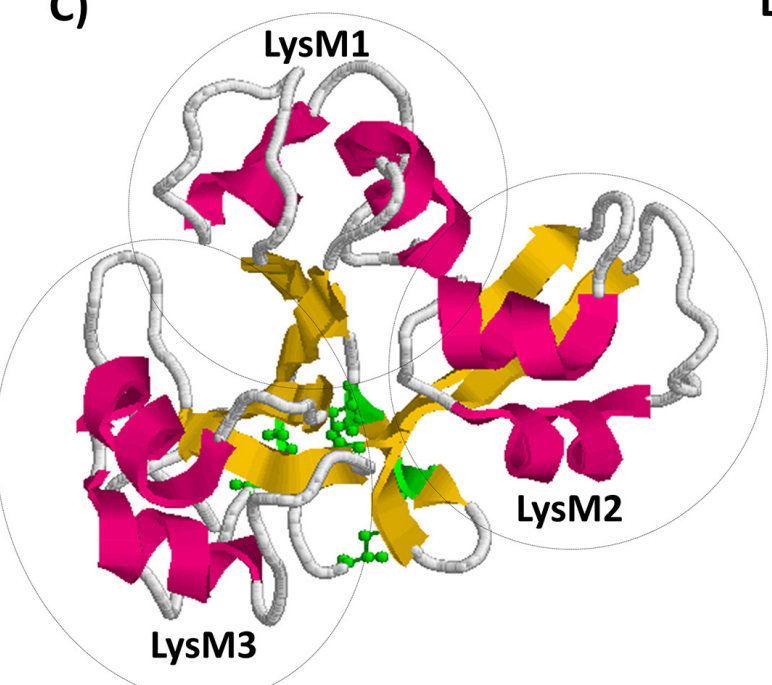

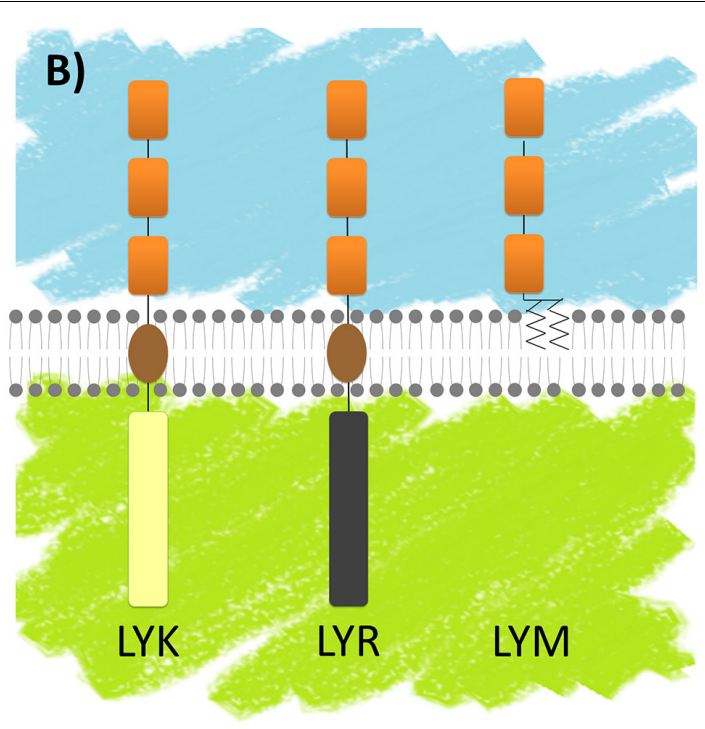

D)

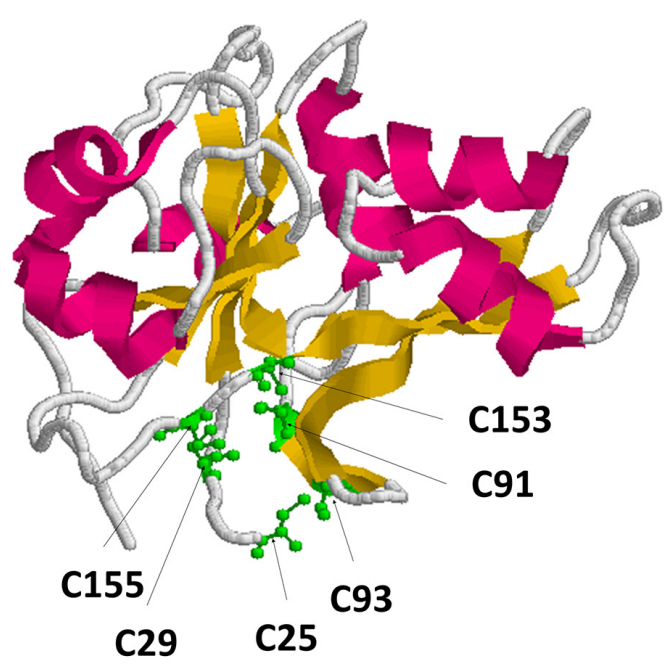

FIGURE 1 | LysM-RLK and LysM-RLP structure, synthesis and maturation. (A) Receptor-like kinases (RLKs) are produced by ribosomes associated with the ER. The ECR that is preceded by a SP is translocated into the ER lumen during translation until the TM (brown) is inserted in the lipid bilayer. The ICR is then produced in the cytosol. SPs are cleaved in the ER and the mature proteins are transported through the secretory pathway to their final destinations, mainly the plasma membrane. GPI anchored receptor-like proteins (RLPS) are also produced by ribosomes associated with the ER. After translocation and insertion in the membrane, the SP is cleaved and the ECR is transferred to a GPI anchor. The mature proteins are then transported to their final destinations. (B) LysM-RLKs are composed of 3 lysin motifs (LysM, orange) in the ECR, a TM (brown) and an ICR bearing an active kinase (beige, LYK subfamily) or an inactive kinase (gray, LYR subfamily). LysM-RLPs (LYMs) are composed of 3 LysMs in the ECR attached to a GPI anchor. (C,D) AtCERK1 3D structure resolved by Liu T. et al. (2012). Images were obtained using the pdb file 4EBY: $\alpha$-helices are indicated in pink, $\beta$-strands are indicated in yellow and $\mathrm{C}$ residues are indicated in green. (C) Orientation of AtCERK1 ECR highlighting the 3 LysMs (circled) packed together. (D) Orientation of AtCERK1 ECR highlighting the $\mathrm{C}$ residues involved in disulfide bridges.

In their ICRs, plant RLKs have a domain that has homology to the serine/threonine kinases. However, it was shown that in addition to phosphorylation of S/T residues, several plant RLKs can phosphorylate tyrosine residues ( $\mathrm{Oh}$ et al., 2009; KlausHeisen et al., 2011). The canonical form contains a catalytic D residue preceded by an $\mathrm{R}$ and is called an $\mathrm{RD}$ kinase. A variant kinase domain found in plant RLKs lacks the R preceding the catalytic $\mathrm{D}$ and for this reason is called a non- $\mathrm{RD}$ kinase. Several non-RD kinases appear to have relatively weak kinase activity in vitro compared to the RD kinases, and their kinase activity is partially dispensable for their function (For review, Schwessinger and Ronald, 2012). Kinase domains contain other essential conserved features among which a G-rich loop involved in nucleotide binding. Other variant kinase domains found in 
plant RLKs lack conserved features such as the G-rich loop and do not exhibit auto-phosphorylation activity in vitro. The latter are called dead-kinases or pseudo-kinases.

Another family of plant proteins possess ECRs similar to those of RLKs but lacks ICRs. Among these proteins, called RLPs, some contain only the ECR and are soluble while others are anchored in membrane either with through a TM or a GPI anchor (Figure 1A).

Plant RLKs and GPI-anchored RLPs are mainly found at the PM, although they transiently accumulate in internal compartments of the secretory or endocytic pathways during their life cycle. Indeed, as integral PM proteins, they are produced at the ER. RLKs are type I transmembrane proteins. They bear a SP at their N-terminus (Figure 1A) allowing translocation of the ECR in the ER lumen during protein synthesis. SP is then cleaved, TM is embedded in the ER membrane and the RLKs follow the secretory pathway to the PM. From the PM they can be internalized at the end of their life cycle or after ligand perception through the endocytic pathway and they are ultimately degraded in the lytic vacuole (for review, Beck et al., 2012).

\section{Lysin Motif Receptor-Like Kinases}

Lysin motif receptor-like kinases (LysM-RLKs) and lysin motif receptor-like proteins (LysM-RLPs) are subfamilies of plant RLK/Ps that contain three LysMs in their ECR (Figure 1B). A LysM is a protein domain of about 40 AA found in most living organisms except in Archaea (Buist et al., 2008). Its name originates from its identification in bacterial autolysin proteins that hydrolyze bacterial PGN and lead to cell lysis. Although not highly conserved in terms of primary sequence, LysMs have highly conserved secondary and tertiary structures consisting of two $\alpha$-helices stacking onto two antiparallel $\beta$-sheets as determined by NMR spectroscopy or X-Ray crystallography (Bateman and Bycroft, 2000; Bielnicki et al., 2006; Liu T. et al., 2012; Maxwell et al., 2013; Sánchez-Vallet et al., 2013; Mesnage et al., 2014; Wong et al., 2014; Koharudin et al., 2015; Leo et al., 2015; Liu et al., 2016). Highly conserved C pairs separated by one AA (CXC) are found between the LysMs of all plant LysM-RLK/Ps. These C pairs are involved in disulfide bridges (Lefebvre et al., 2012; Liu T. et al., 2012; Liu et al., 2016) that pack the three LysMs together (Figures 1C,D).

Two main types of plant LysM-RLKs can be defined based on their kinase domains (Figure 1B). The first type, named LYK (Limpens et al., 2003), has a canonical RD kinase and shows in vitro autophosphorylation activities (Petutschnig et al., 2010; Klaus-Heisen et al., 2011; Madsen et al., 2011; Zeng et al., 2012). The second type, named LYR (Arrighi et al., 2006), carries an aberrant kinase lacking some conserved features such as the G-rich loop, and does not exhibit either auto-phosphorylation or trans-phosphorylation activities when tested in vitro (Arrighi et al., 2006; Madsen et al., 2011). GPI-anchored LysM-RLPs are also found in plants and named LYMs (Arrighi et al., 2006).

Most of the LysM-RLK/Ps that have been studied were shown to perceive structurally related GlcNAc containing molecules and/or to be involved in plant-microbe interactions including establishment of defense responses or root endosymbioses. In this review, we report the currently known biological roles and biochemical functions of plant LysM receptor proteins and discuss conservation or evolution of LysM-RLK/P roles and functions in various phylogenetic groups.

\section{Microbe-Associated Molecular Pattern Triggered Immunity}

One layer of plant defense against pathogenic microbes involves perception by plants of conserved microbial signatures also called MAMPs, and consequently induction of MTI. MTI mainly consists in basal defense mechanisms such as cell wall reinforcement, stomatal closure and synthesis of antimicrobial compounds that can lead in some conditions to cell death. Many plant RLKs are involved in MAMP perception and signaling (for review, Schwessinger and Ronald, 2012). Because MAMPs are conserved microbial signatures, they are not specific to pathogens but are also present in beneficial microbes. Specific signatures can also be perceived by plants. In most cases, these specific signatures are proteins called effectors. Effectors are secreted by microbes to manipulate plant signaling, defense or metabolism and the effector repertoire is highly variable within microbial species. Recognition of such proteins produced by pathogens can induce ETI that in most cases leads to cell death.

Plant treatment with various MAMPs typically induces similar responses (such as alkalinisation of the extracellular medium, ROS production, MAP kinase phosphorylation and induction of defense-related gene transcription). These responses have been used to identify and characterize MAMPs. Chitin fragments are typical fungal MAMPs. Chitin is a long-chain $\beta-1,4$ GlcNAc polymer, which is the major component of fungal cell walls. Although chitin is insoluble, COs are GlcNAc oligomers (Figure 2), soluble at least up to a degree of polymerization of 8 GlcNAc residues. COs can be produced by chitin cleavage through the action of plant secreted chitinases. Chitin and COs are sometimes used indiscriminately in the literature leading to confusion. For this reason, here we refer to chitin as long insoluble polymers and we mention the degree of polymerization of $\mathrm{CO}$ (i.e., CO8 for 8 GlcNAc oligomers). CO8 has been shown to be the most active oligomer among COs for activation of defense-related responses (Kuchitsu et al., 1997). PGN fragments are typical bacterial MAMPs. PGN is a major component of bacterial cell walls. It is a polymer of alternating GlcNAc and $N$-acetylmuramic acid residues, branched with AAs. Like chitin, PGN is insoluble, while muropeptides (Figure 2) are soluble PGN fragments. Chitin and PGN fragments are both perceived by LysM-RLK/Ps as detailed below.

Many other MAMPs do not contain GlcNAc. One of the best characterized bacterial MAMPs is flagellin. Various flagellin peptides are perceived by RLKs in animals and plants (for review, Fliegmann and Felix, 2016). The flg22 peptide (originally identified in Pseudomonas aeruginosa; Felix et al., 1999) is perceived in Arabidopsis thaliana by AtFLS2, a leucine rich repeat receptor-like kinase (LRR-RLK, Gomez-Gomez and Boller, 2000; Chinchilla et al., 2006). Another well characterized bacterial MAMP is the elf18 peptide found in the bacterial elongation factor (EF-Tu) which is perceived in A. thaliana by another LRR-RLK called AtEFR (Zipfel et al., 2006). 


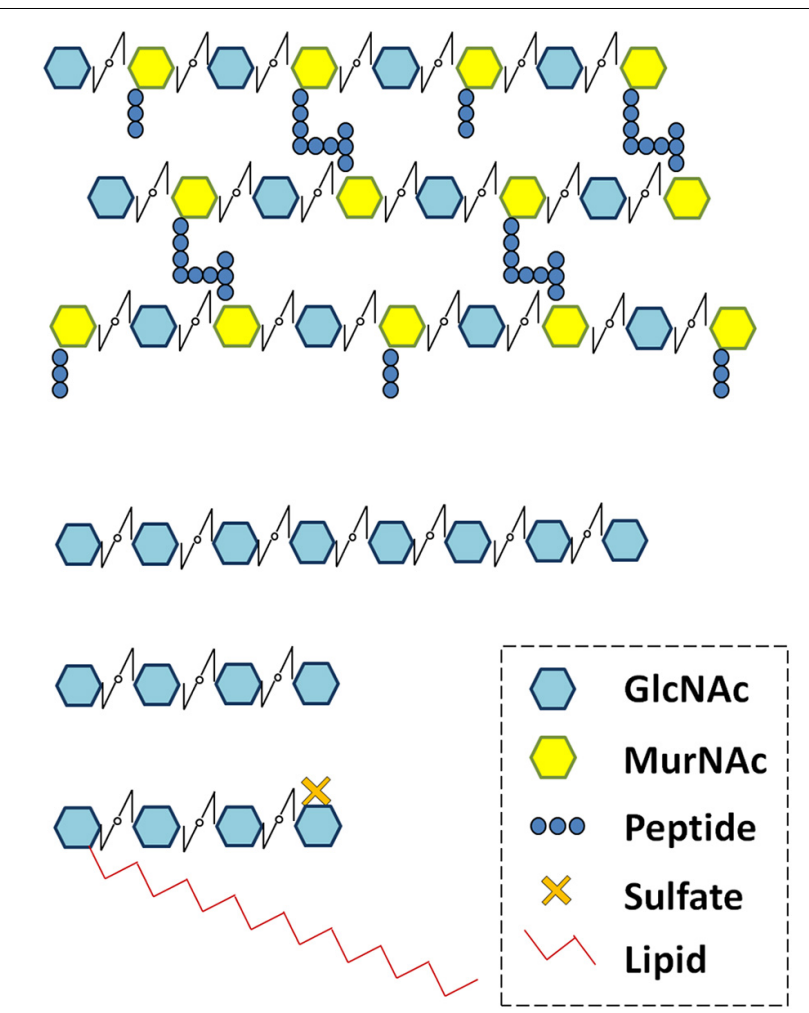

FIGURE 2 | Peptidoglycan (PGN), chitooligosaccharides (CO4 and CO8) and lipo-chitooligosaccharide (LCO-IV) schematic structures. Schematic structures of various $\mathrm{N}$-acetyl glucosamine (GlcNAc) containing molecules produced by microorganisms. GlcNAc residues are associated to $\mathrm{N}$-acetylmuramic acid (MurNAc) in PGN and to lipid in LCOs.

\section{Root Endosymbioses}

Plants also interact with many beneficial microbes. AMF can colonize the roots of most terrestrial plants, by establishing an extended hyphal network in the soil and by providing plants with mineral nutrients collected in the soil. Nitrogen fixing bacteria called Rhizobia and Frankia are able to trigger the formation of particular plant root organs called nodules, in phylogenetically related legumes and actinorhizal plants, respectively. Inside nodules, these bacteria can efficiently reduce gaseous atmospheric nitrogen $\left(\mathrm{N}_{2}\right)$ to ammonia $\left(\mathrm{NH}_{3}\right)$ and hence provide a nitrogen source to plants. For this reason, these bacterial genera are extremely important for plant nutrition. Despite the differences in the nature of the microorganisms involved, the AMS and the RNS share commonalities. The mechanism of RNS establishment is considered to originate from the more ancient AMS. Notably, plant genes that control a signaling pathway, called the CSSP, are required for establishment of both AMS and RNS. CSSP activation leads to the production and decoding of oscillations in the calcium concentration (also called calcium spiking) in and around plant cell nuclei. Genes that code for all the components of the CSSP are only found in plants that can establish at least one of these symbioses (Delaux et al., 2014). In such plants, mutations in CSSP genes lead to an absence of AMF penetration at the root epidermis (for review, Gutjahr and Parniske, 2013).
In legume and actinorhizal plants, mutations in CSSP genes also block nodule development and bacterial colonization of plant roots (for review, Svistoonoff et al., 2014).

The Nod-factors are well known Rhizobial secreted molecules essential for bacterial recognition by legumes and subsequently for Rhizobial root colonization. Nod-factors are LCOs composed of a core structure of 4 or 5 GlcNAc residues in which the terminal non-reducing sugar is substituted with an acyl chain (Figure 2). In addition, Rhizobial LCOs bear other substitutions that are characteristic of bacterial strains and important for host specificity (Fliegmann and Bono, 2015). AMF also secrete LCOs similar to those produced by Rhizobia (Maillet et al., 2011), as well as short-chain $\mathrm{CO}$ ( $\mathrm{CO} 4$ and $\mathrm{CO} 5$, Figure 2, Genre et al., 2013) that might correspond to LCO precursors. Exogenous application of LCOs or short-chain COs activates plant responses such as extracellular medium alkalinization (Staehelin et al., 1994; Felle et al., 1996), calcium spiking (Oldroyd et al., 2001; Harris et al., 2003; Sun et al., 2015), or promotion of lateral root development (Olah et al., 2005; Sun et al., 2015; Herrbach et al., 2017) in various plant species including nodulating and non-nodulating plants. These plant responses have been shown to be CSSP-dependent. In addition, regulation of symbiosis-related gene transcription by treatment with LCOs has been shown in legumes (Combier et al., 2008; Camps et al., 2015; Hohnjec et al., 2015) but not yet reported in non-legumes. The $\mathrm{CO} 4$ and $\mathrm{CO} 5$ produced by AMF are referred as Myc-COs or short-chain COs and are currently considered to play a role in AMS establishment in contrast to the long-chain $\mathrm{CO}, \mathrm{CO} 7$ and $\mathrm{CO} 8$ described to be defense elicitors. Effectors are also produced by AMF (Kloppholz et al., 2011; Kamel et al., 2017) and some rhizobia (Deakin and Broughton, 2009; Yang et al., 2010; Okazaki et al., 2013), and can be involved in symbiosis establishment.

\section{PHYLOGENETIC ANALYSIS}

\section{Methodology}

Studies that deal with functional characterization of LysM-RLK/Ps have been performed only within a few species that represent the genetic diversity of higher plants. This includes dicotyledons (A. thaliana, Medicago truncatula, Lotus japonicus, and Solanum lycopersicum) and a monocotyledon (Oryza sativa). Although several LysM-RLK/P phylogenetic trees have been published (Arrighi et al., 2006; Zhang et al., 2009; Lohmann et al., 2010; Shimizu et al., 2010; Zeng et al., 2012; Buendia et al., 2016; Bono et al., 2018) none of these phylogenies include all the listed species at the same time. Moreover naming of LysM-RLK/Ps has been done independently in each species making the comparison between species complicated.

To discuss the evolution of LysM-RLK/Ps in higher plants, we have inferred phylogenetic trees using phyML (Figures 3, 4, 6) and MrBayes (Supplementary Figures S1-S4). In addition to the species mentioned above, we used the sequences of two additional dicotyledons (Prunus persica and Brassica rapa) and one more monocotyledon (Brachypodium distachyon) in which the genome sequences have been published. We performed manual correction of many gene structure predictions (see 
notes in Supplementary Table S1). Phylogenetic trees were inferred independently with predicted protein sequences of the LYMs (Figure 3 and Supplementary Figure S1), LYRs (Figure 4 and Supplementary Figure S2) or LYKs (Figure 6 and Supplementary Figure S3). Some proteins identified as putative LysM-RLK/Ps were not used for the phylogenetic analysis because they were truncated or their sequence/existence was uncertain (these proteins are indicated in italics in Supplementary Table S1). We focused our analysis on the membrane-anchored LysM-RLPs (LYMs) and we did not consider the soluble LysM-RLPs containing 3 LysMs (called LYP clade II in Zhang et al., 2009). We used PredGPI ${ }^{1}$ to identify the GPI anchor sites in the LYMs. Orthologous genes based on the phylogenetic trees are arranged in Supplementary Table S1 in lanes with a color code. To reinforce ortholog identification, we reported the intron-exon structure in Supplementary Table S1 which is almost conserved among all the orthologs. Minor

\footnotetext{
${ }^{1}$ http://gpcr.biocomp.unibo.it/predgpi/pred.htm
}

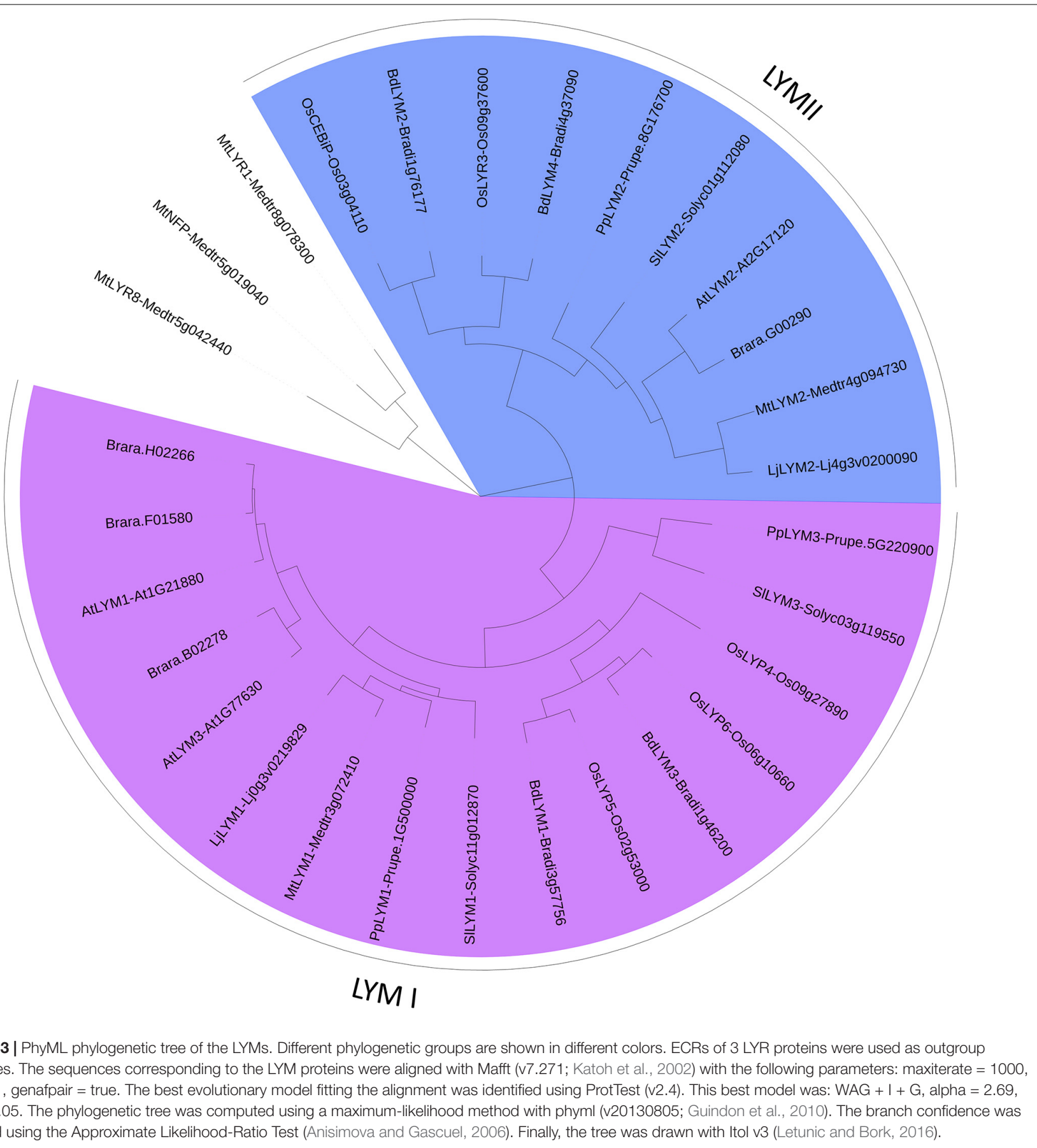




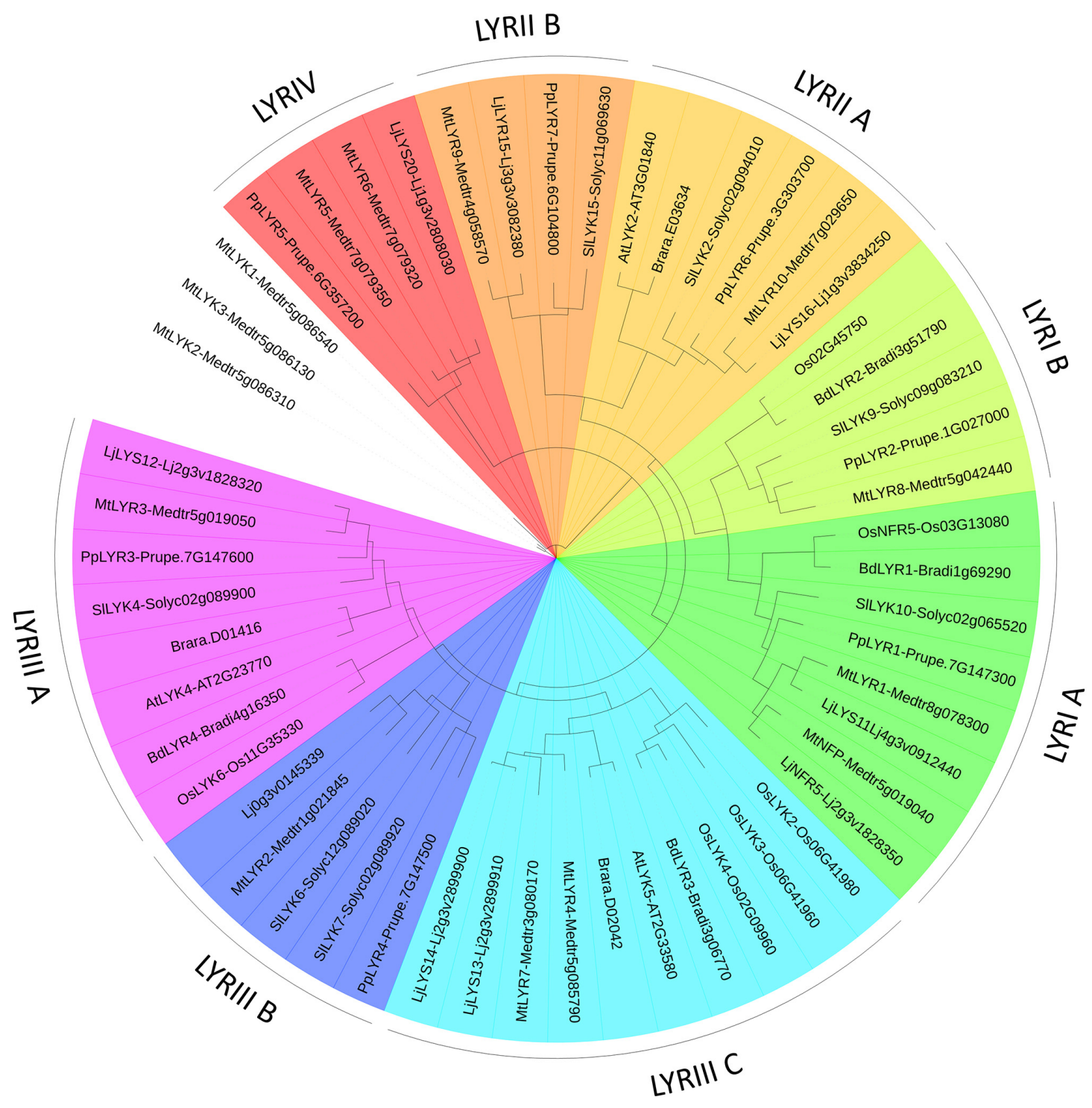

FIGURE 4 | PhyML phylogenetic tree of the LYRs. Different phylogenetic groups are shown in different colors. Three LYK proteins were used as outgroup sequences. The same protocol as for the LYM family tree (Figure 3) was used, except that the best model fitting the alignment was $L G+I+G, a l p h a=1.71, p-i n v=0.06$.

differences might be due to evolutionary changes or to residual errors in gene structure predictions.

We found a high variability in the number of LysM-RLKs (5-22) and membrane-anchored LysM-RLPs (2-5) between the eight plant species analyzed, with an expansion of the LysM-RLKs in dicotyledons (except in the Brassicaceae) versus monocotyledons and an expansion of membrane-anchored LysM-RLPs in monocotyledons versus dicotyledons. Legumes showed the highest number of LysM-RLKs and the lowest number of membrane-anchored LysM-RLPs. Although the number of genes is highly variable, phylogenetic groups can be distinguished with members in almost all species. We distinguished 2 phylogenetic groups of LYMs (Figure 3 and Table 1), 2 phylogenetic groups of LYRs (Figure 4), and 3 phylogenetic groups of LYKs (Figure 6) common to dicotyledons and monocotyledons. Two additional phylogenetic groups of LYRs and several subgroups were found only in dicotyledons. We propose to name the phylogenetic groups in the trees as LYM, LYR, and LYK with one number, and a letter when subgroups can be distinguished (Supplementary Table S1). Below and in Supplementary Table S1, we also reported the nomenclature proposed by Zhang et al. (2009). Most phylogenetic subgroups have one member in all species with few exceptions of duplications in particular species. Two phylogenetic groups (LYRI and LYKI) have, however, encountered many duplication events that explain most of the variability in the number of LysM-RLKs between species.

Almost no introns were found in the $5^{\prime}$ part of the genes encoding the 3 LysMs, in either LYRs, LYKs, or LYMs. In contrast, strong differences of intron number were observed 


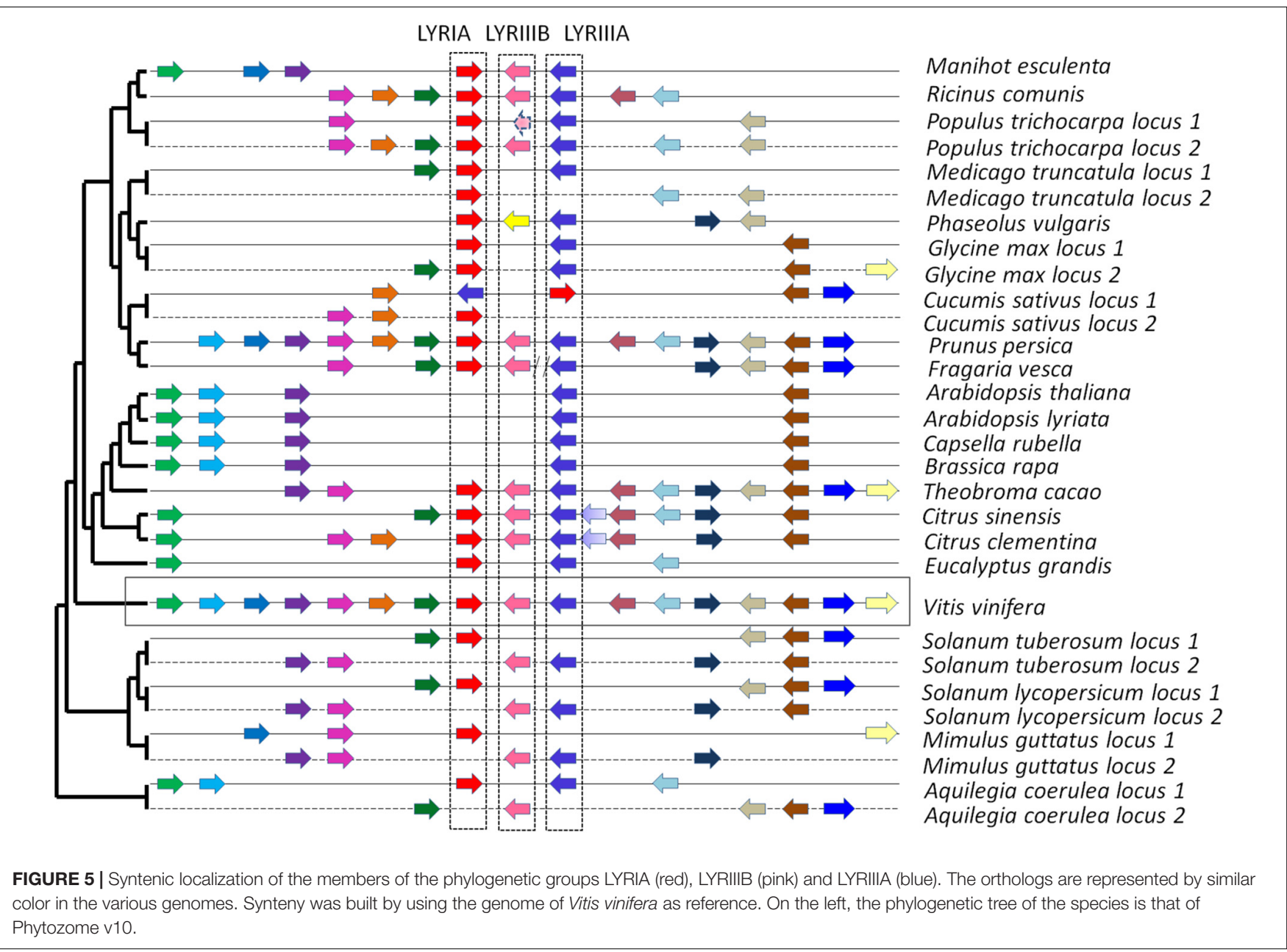

in the sequence encoding the extracellular juxta membrane region and the ICR (0/1 in LYRs, 3/4 in LYMs and 9/12 in LYKs). This suggests independent combinations of the sequence encoding the 3 LysMs with either one encoding a dead kinase for the LYR ancestor, one encoding an active kinase for the LYK ancestor and one encoding a site for GPI anchoring for the LYM ancestor. This might have happened at an early time of plant evolution. Indeed, members of the phylogenetic groups LYRI and LYKI can be found in more ancient plant genera (Zhang et al., 2009) such as Physcomitrella (bryophytes) and Selaginella (lycophytes).

Biological roles and biochemical functions, as well as the evolution of the number of members for each phylogenetic group are discussed below.

\section{Description of the Phylogenetic Groups LysM-RLP LYMI (LYP Clade I in Zhang et al., 2009)}

One to three members can be found in the phylogenetic group LYMI (Figure 3, Table 1, and Supplementary Figure S1). Studies concerning members of this phylogenetic group have only been performed in A. thaliana and $O$. sativa. They are involved in perception of $\mathrm{PGN}$ and resistance to bacterial pathogens.
In $A$. thaliana, the two members of this phylogenetic group, AtLYM1 (At1g21880) and AtLYM3 (At1g77630) are required for activation of PGN signaling (Willmann et al., 2011). In Atlym1 or Atlym3 knock-out mutants, transcriptional responses to PGN are abolished and growth of the pathogenic bacterial strain Pseudomonas syringae pv. tomato DC3000 is increased. The double mutant has a similar phenotype to single mutants suggesting a cooperative role of the two proteins rather than a redundant role. These two genes do not play a role in chitin perception. To demonstrate PGN binding, AtLYM1 and AtLYM3 ECRs were produced in Escherichia coli, purified and pulleddown using insoluble PGN. Attachment of AtLYM1 or AtLYM3 ECR to insoluble PGN decreased in the presence of soluble PGN fragments (PGN hydrolyzed by sonication), but not in the presence of CO6, CO8, or LCOs, showing that AtLYM1 and AtLYM3 specifically bind PGN fragments (Willmann et al., 2011). The affinity of AtLYM1 and AtLYM3 for PGN is, however, not known.

In O. sativa, OsLYP4 (Os09g27890) and OsLYP6 (Os06g10660), two of the three members in the phylogenetic group LYMI, have been reported to play a similar role in PGN recognition but surprisingly also in CO recognition (Liu B. et al., 2012). In plants with decreased expression of OsLYP4 or OsLYP6 by RNAi, 


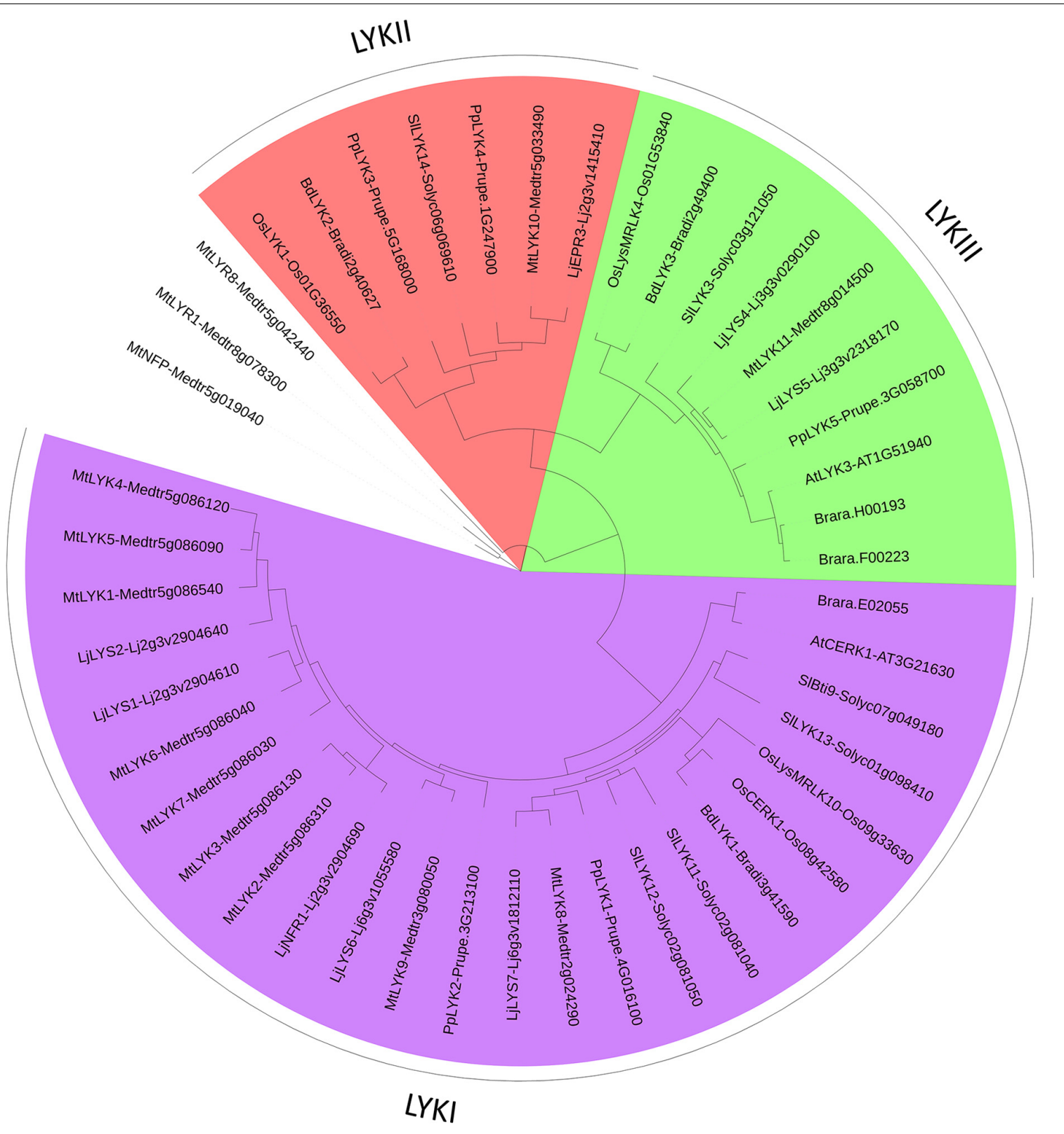

FIGURE 6 | PhyML phylogenetic tree of the LYKs. Different phylogenetic groups are shown in different colors. Three LYR proteins were used as outgroup sequences. The same protocol as for the LYM family tree (Figure 3) was used, except that the best model fitting the alignment was JTT $+1+\mathrm{G}$, alpha $=1.32, p$-inv $=0.1$.

responses to PGNs and to CO6 (transcriptional responses and callose deposition) were decreased and lesions due to bacterial (Xanthomonas oryzae or X. oryzicola) or fungal (Magnaporthe oryzae) pathogens were increased. In contrast, overexpression of the two proteins led to decreased lesions in presence of these pathogens. OsLYP4 and OsLYP6 ECRs were also produced in E. coli, purified and pulled-down using insoluble PGN or insoluble chitin. Competition assays were performed using $\mathrm{CO} 6$ or soluble PGN fragments (PGN hydrolyzed by lysostaphin). Each of these molecules was able to inhibit OsLYP4 and OsLYP6 pull-down either by insoluble chitin or PGN, showing that the same binding site was responsible for CO and PGN fragment perception. The affinity of OsLYP4 and OsLYP6 for PGN or chitin is, however, not known. OsLYP5 (Os02g53000) is also a member of this phylogenetic group and OsLYP5 and OsLYP6 are close paralogs while OsLYP4 is slightly divergent (Figure 3, Liu B. et al., 2012). In contrast to what is mentioned in Liu B. et al. (2012), we found a predicted TM and GPI anchor site in OsLYP5. Using siRNA-Finder $\left(\mathrm{Si}_{-} \mathrm{Fi}^{2}\right)$ we found that the construct used to silence OsLYP6 in Liu B. et al. (2012) is predicted to target OsLYP5 as well. Thus an analysis of OsLYP5 role in PGN and chitin perception and an analysis of cross silencing of OsLYP5 by

\footnotetext{
${ }^{2}$ http://labtools.ipkgatersleben
} 
TABLE 1 | LysM-RLPs belonging to the phylogenetic groups LYMI or LYMII found in the 8 species analyzed.

\begin{tabular}{|c|c|c|c|c|c|c|c|c|}
\hline & M. truncatula & L. japonicus & P. persica & A. thaliana & B. rapa & S. lycopersicum & B. distachyon & O. sativa \\
\hline \multirow[t]{8}{*}{ LYMI } & Medtr3g072410 & Lj0g3v0219829 & Prupe.1G500000 & At1g21880 & Brara.F01580 & Solyc11g012870 & Bradi3g57756 & Os06g10660 \\
\hline & LYM1 & & PpLYM1 & AtLYM1 & & SILYM1 & BdLYM1 & OsLYP6 \\
\hline & & & & & Brara.H02266 & & Bradi1g46200 & Os02g53000 \\
\hline & & & & & & & BdLYM3 & OsLYP5 \\
\hline & & & & At1g77630 & Brara.B02278 & & & Os09g27890 \\
\hline & & & & AtLYM3 & & & & OsLYP4 \\
\hline & & & Prupe.5G220900 & & & Solyc03g119550 & & \\
\hline & & & PpLYM3 & & & SILYM3 & & \\
\hline \multirow[t]{4}{*}{ LYMII } & Medtr4g094730 & Lj4g3v0200090 & Prupe.8G176700 & At2g17120 & Brara.G00290 & Solyc01g112080 & Bradi1g76177 & Os03g04110 \\
\hline & LYM2 & & PpLYM2 & AtLYM2 & & SILYM2 & BdLYM2 & OsCEBiP \\
\hline & & & & & & & Bradi4g37090 & Os09g37600 \\
\hline & & & & & & & BdLYM4 & OsLYP3 \\
\hline
\end{tabular}

OsLYP6 hairpin constructs are required to clarify the differences in the biological role between A. thaliana and O. sativa orthologs.

LysM-RLP LYMII (LYP Clade III in Zhang et al., 2009)

In dicotyledons, only one member was found in this phylogenetic group, while two members were present in monocotyledons (Figure 3, Table 1, and Supplementary Figure S1). Members of this phylogenetic group and especially rice OsCEBIP are among the best characterized LysM-RLK/Ps. They are involved in long-chain $\mathrm{CO}$ perception and resistance to fungal pathogens.

In rice, knock-down of Chitin Elicitor Binding Protein (OsCEBiP, Os09g37600) by RNAi resulted in a decrease of the CO8-induced oxidative burst in a rice cell culture (Kaku et al., 2006) and in an increase of $M$. oryzae colonization in rice plants (Kishimoto et al., 2010). On the contrary, OsCEBiP overexpression led to a decrease of lesion size by $M$. oryzae. Its role as a main actor in $\mathrm{CO} 8$ perception was further confirmed in cell cultures derived from knock-out plants (Kouzai et al., 2014b). Although OsCEBIP was firstly described to have 2 LysMs, elucidation of its 3D structure (Liu et al., 2016) unambiguously demonstrated that it bears 3 LysMs as all LysM-RLK/Ps. OsCEBiP was originally purified from rice cell cultures (Kaku et al., 2006) in which a binding site for CO8 had been characterized using a radiolabeled $\mathrm{CO} 8$ derivative with $K d$ of 5.4 or $29 \mathrm{nM}$ in microsomal fraction (Shibuya et al., 1993) or PM fraction (Shibuya et al., 1996), respectively. Similarly, half maximal incorporation of a radiolabeled photoactivatable $\mathrm{CO} 8$ derivative in microsomal fractions of a rice cell culture was about $50 \mathrm{nM}$ (Ito et al., 1997). Using a biotinylated CO8 derivative that can be crosslinked to proteins and detected by Western blotting (with antibodies raised against biotin), it has been shown that the $\mathrm{CO} 8$ binding site detected in rice cell culture disappears when OsCEBIP is silenced (Shinya et al., 2010) or knocked-out (Kouzai et al., 2014b). OsCEBIP was also expressed heterologously in a tobacco BY-2 cell culture and half saturation was found around $100 \mathrm{nM}$ using the biotinylated CO8 derivative (Shinya et al., 2012). As shown for the CO8 binding site in rice cell cultures (Shibuya et al., 1996), competition assays with different lengths of CO on OsCEBIP expressed in BY-2 cells demonstrated higher affinity for CO8 than for shorter COs (Shinya et al., 2010,
2012). Note that all CO8 derivatives used in these studies have an opened GlcNAc at the reducing-end and were shown to have biological activities lower than $\mathrm{CO} 8$ and comparable to CO7. However, this modification does not affect the affinity deduced from the competition assays with unmodified COs. More recently, OsCEBIP ECR was expressed in insect cells and purified. Affinities of $3 \mu \mathrm{M}$ for $\mathrm{CO} 4$ and $4 \mu \mathrm{M}$ for $\mathrm{CO} 8$ were determined by ITC (Liu et al., 2016). The CO binding site was found on the second LysM both by NMR spectroscopy and modeling (Hayafune et al., 2014) and by X-Ray crystallography (Liu et al., 2016). By mutating I150 in LysM2 [named 122 in Hayafune et al. (2014) as numbering started after the SP], it was demonstrated that this residue is critical for $\mathrm{CO}$ binding (Hayafune et al., 2014; Liu et al., 2016). Because the binding site on the second LysM is occupied by a CO3 (Hayafune et al., 2014; Liu et al., 2016), it has been hypothesized that CO8 binding occurs through dimerization of OsCEBIP (Hayafune et al., 2014; Liu et al., 2016). CO8 was actually found to induce in vitro dimerization of the OsCEBIP ECR produced in E. coli (Hayafune et al., 2014), but not of the OsCEBIP ECR produced in insect cells (Liu et al., 2016). It has been also shown that OsCEBIP ECR is able to form homodimers in a yeast two hybrid system and that part of the OsCEBIP is found at a size corresponding to homodimer in vivo in absence of COs using blue native polyacrylamide gel electrophoresis and immunodetection (Shimizu et al., 2010). The requirement of dimerization to form a high affinity binding site might explain the low affinity for $\mathrm{CO} 8$ found in the OsCEBIP ECR produced in insect cells (Liu et al., 2016) and its inability to discriminate CO4 and CO8 (Liu et al., 2016), in contrast to the previous OsCEBIP biochemical characterization. Interestingly $100 \mathrm{nM}$ of (GlcN $\beta 1,4 \mathrm{GlcNAc})_{4}$, an oligosaccharide alternating $N$-acetylated and non- $\mathrm{N}$-acetylated glucosamine (therefore carrying $\mathrm{N}$-acetyl moieties only on one face of the polymer) was shown to inhibit CO8-induced OsCEBIP in vitro dimerization and ROS production in rice cells (Hayafune et al., 2014). In contrast, $100 \mathrm{nM} \mathrm{CO} 4$ did not compete CO8 for these responses (Hayafune et al., 2014). This suggests that two OsCEBIP monomers bind a single $\mathrm{CO} 8$ molecule, with each OsCEBIP monomer binding to an opposite face and side of the $\mathrm{CO} 8$ molecule. 
In wheat and barley, orthologs of OsCEBIP were also shown to be involved in defense against pathogens. Wheat lines that were knockdown for TaCEBIP by VIGS showed disease symptoms produced by the fungal pathogen Mycosphaerella graminicola line (mutated for an effector involved in virulence) which was reported not to be pathogenic on WT wheat plants (Lee et al., 2014). Barley lines knock-down for HvCEBIP by VIGS also showed increased lesions due to M. oryzae (Tanaka et al., 2010). In WT lines of these plant species, $\mathrm{CO} 8$ binding sites similar to those of rice were detected (Okada et al., 2002) although the corresponding proteins have not been characterized.

In $A$. thaliana, the only member of the phylogenetic group LYMII, AtLYM2 (At2g17120) is also a chitin-binding protein. Expressed in BY-2 cells, AtLYM2 showed binding to COs as OsCEBIP (Shinya et al., 2012). Surprisingly, AtLYM2 is not required for general responses to COs (Shinya et al., 2012; Narusaka et al., 2013). However, AtLYM2 was reported to be involved in defense against the fungal pathogens Botrytis cinerea and Alternaria brassicicola (Faulkner et al., 2013; Narusaka et al., 2013). This might occur through control of symplastic fluxes in response to COs (Faulkner et al., 2013).

In $M$. truncatula, the only member of the phylogenetic group LYMII, MtLYM2 (Medtr4g094730) was expressed in BY-2 cells and was reported to bind long-chain COs (Fliegmann et al., 2011) but its affinity and involvement in $\mathrm{CO}$ responses have not been characterized.

Although all orthologs of OsCEBIP studied so far seem to have similar $\mathrm{CO}$ binding properties and to be involved in basal resistance to pathogenic fungi, they appear to be involved in various mechanisms. These mechanisms have been characterized only in rice and Arabidopsis. Studies in additional plant species are required to determine whether OsCEBIP orthologs are involved in defense mechanisms similarly to those found in rice or those found in Arabidopsis. Moreover the role of the second member of the phylogenetic group LYMII in monocotyledons needs to be determined.

\section{LysM-RLK LYRI (LYK Clade I in Zhang et al., 2009)}

Although absent in $A$. thaliana and B. rapa, all the plant species analyzed here have at least two members in the phylogenetic group LYRI, which can be divided into two subgroups here called $\mathrm{A}$ and $\mathrm{B}$, each of them containing in most cases one member (Figure 4, Table 2, and Supplementary Figure S2). Some legumes have the particularity to possess two genes in the subgroup A (Gough and Jacquet, 2013). Members of the phylogenetic group LYRIA and especially the legume MtNFP (Medtr5g019040)/LjNFR5 (Lj2g3v1828350) are also among the best-characterized LysM-RLK/Ps. They control Nod-factor perception and establishment of root endosymbioses.

\section{Subgroup LYRIA}

MtNFP and LjNFR5 are required for the RNS in M. truncatula (Arrighi et al., 2006) and L. japonicus (Madsen et al., 2003), respectively. Almost no LCO response is detected in plants mutated in MtNFP or LjNFR5. Knockdown of MtNFP together with expression data suggest that it is involved in perception of Rhizobia all along the colonization process, including within nodules (Arrighi et al., 2006). Surprisingly, plants mutated in MtNFP have also been shown to be more sensitive to the pathogenic oomycetes Aphanomyces euteiches (Rey et al., 2013) and Phytophthora palmivora (Rey et al., 2015) and the fungal pathogens Colletotrichum trifolii (Rey et al., 2013) and Verticillium albo-atrum (Ben et al., 2013). Affinity for LCOs has been reported for LjNFR5 (Broghammer et al., 2012). LjNFR5 was expressed in a heterologous plant system (leaves of Nicotiana benthamiana), solubilized and purified to determine its affinity for an LCO with a structure close to the main Nodfactor produced by Mesorhizobium loti (the symbiotic partner of L. japonicus in RNS). High affinity was measured by SPR and MST, with a $K d$ of 4 and $10 \mathrm{nM}$, respectively. For SPR, an LCO derivative was immobilized on a chip and affinity determined using a range of LjNFR5 concentrations. For MST, affinity was determined using a fluorescent LCO derivative and a range of protein concentrations.

Whereas MtNFP and LjNFR5 are not essential for establishment of AMS in legumes (Ben Amor et al., 2003; Madsen et al., 2003), the tomato ortholog, SlLYK10 (Solyc02g065520) plays a role in AMS establishment (Buendia et al., 2016). Plants with decreased SILYK10 expression by VIGS showed a delay and less efficient colonization by the AMF Rhizophagus irregularis. In contrast, a rice knock-out mutant in the orthologous gene, OsNFR5 (Os03G13080), was normally colonized by R. irregularis compared to WT plants although expression of AMS plant marker genes were reduced in the Osnfr5 mutant compared to the WT, indicating a possible but weak role of OsNFR5 in AMS establishment. Moreover, a chimera consisting of the sequences encoding LjNFR5 ECR and OsNFR5 ICR was able to complement the absence of nodulation in a Ljnfr5 mutant, indicating that the function of the ICR is conserved between LjNFR5 and OsNFR5 (Miyata et al., 2016). Interestingly, in Parasponia andersonii, which belongs to a unique group of non-legume species able to form both the RNS with Rhizobia and the AMS, two members, resulting from a tandem duplication, are found in the phylogenetic group LYRIA (van Velzen et al., 2018). PaNFP2 is closest to orthologs in legumes while PaNFP1 is closest to orthologs in non-nodulating species. In non-nodulating species closely related to $P$. andersonii such as Trema species and in $P$. persica, the PaNFP2 orthologs are truncated likely leading to non-functional proteins (van Velzen et al., 2018; Supplementary Table S1). P. andersonii plants containing a RNAi construct that might target the two paralogs, were affected in establishment of both RNS and AMS (Op den Camp et al., 2011). This suggests that an ancestral gene involved in LCO perception and AMS establishment was duplicated before the apparition of nodulation. One copy was then recruited during evolution for LCO perception in RNS establishment at least in legumes and $P$. andersonii. In some legumes, a second member of the phylogenetic group LYRIA is also found. MtLYR1 (Medtr8g078300) in M. truncatula and LjLYS11 (Lj4g3v0912440) in L. japonicus are the paralogs of MtNFP and LjNFR5, respectively. MtLYR1 transcripts are detected in roots but not in nodules (Arrighi et al., 2006). During the AMS, $M t L Y R 1$ transcripts increased in roots and more particularly cortical cells colonized by AMF (Gomez and Harrison, 2009). 
TABLE 2 | LysM-RLKs belonging to the phylogenetic groups LYRI, LYRII, LYRIII or LYRIV found in the 8 species analyzed.

\begin{tabular}{|c|c|c|c|c|c|c|c|c|c|}
\hline & & M. truncatula & L. japonicus & P. persica & A. thaliana & B. rapa & S. lycopersicum & B. distachyon & O. sativa \\
\hline \multirow[t]{6}{*}{ LYRI } & $A$ & Medtr5g019040 & Lj2g3v1828350 & Prupe.7G147300 & & & Solyc02g065520 & Bradi1g69290 & Os03G13080 \\
\hline & & MtNFP & LjNFR5 & PpLYR1 & & & SILYK10 & Bd LYR1 & OsNFR5 \\
\hline & & Medtr8g078300 & Lj4g3v0912440 & & & & & & \\
\hline & & MtLYR1 & LjLYS11 & & & & & & \\
\hline & $\mathrm{B}$ & Medtr5G042440 & $*$ & Prupe.1G027000 & & & Solyc09g083210 & Bradi3g51790 & Os02G45750 \\
\hline & & MtLYR8 & & PpLYR2 & & & SILYK9 & Bd LYR2 & \\
\hline \multirow[t]{4}{*}{ LYR\|I } & A & Medtr7g029650 & Lj1g3v3834250 & Prupe.3G303700 & At3g01840 & Brara.E03634 & Solyc02g094010 & & \\
\hline & & MtLYR10 & LjLYS16 & PpLYR6 & AtLYK2 & & SILYK2 & & \\
\hline & B & Medtr4g058570 & Lj3g3v3082380 & Prupe.6G104800 & & & Solyc11g069630 & & \\
\hline & & MtLYR9 & LjLYS15 & PpLYR7 & & & SILYK15 & & \\
\hline \multirow[t]{12}{*}{ LYRIII } & A & Medtr5g019050 & Lj2g3v1828320 & Prupe.7G147600 & At2g23770 & Brara.D01416 & Solyc02g089900 & Bradi4g16350 & Os11G35330 \\
\hline & & MtLYR3 & LjLYS12 & PpLYR3 & AtLYK4 & & SILYK4 & Bd LYR4 & OsLYK6 \\
\hline & $\mathrm{B}$ & Medtr1g021845 & Lj0g3v0145339 & Prupe.7G147500 & & & Solyc02g089920 & & \\
\hline & & MtLYR2 & & PpLYR4 & & & SILYK7 & & \\
\hline & & & & & & & Solyc12g089020 & & \\
\hline & & & & & & & SILYK6 & & \\
\hline & C & Medtr5g085790 & Lj2g3v2899910 & & At2g33580 & Brara.D02042 & & Bradi3g06770 & Os06G41960 \\
\hline & & MtLYR4 & LjLYS13 & & AtLYK5 & & & Bd LYR3 & OsLYK3 \\
\hline & & Medtr3g080170 & Lj2g3v2899900 & & & & & & Os06G41980 \\
\hline & & MtLYR7 & LjLYS14 & & & & & & OsLYK2 \\
\hline & & & & & & & & & Os02G09960 \\
\hline & & & & & & & & & OsLYK4 \\
\hline \multirow[t]{4}{*}{ LYRIV } & & Medtr7g079350 & $*$ & & & & & & \\
\hline & & MtLYR5 & & & & & & & \\
\hline & & Medtr7g079320 & Lj1g3v2808030 & Prupe.6G357200 & & & & & \\
\hline & & MtLYR6 & LjLYS20 & PpLYR5 & & & & & \\
\hline
\end{tabular}

*Evidences for their existence (see Supplementary Table S1).

In L. japonicus, LjLYS11 expression was not detected in roots and nodules but in cortical cells colonized by AMF. The expression patterns of MtLYR1 and LjLYS11 suggest a role in the AMS, possibly redundant with MtNFP and LjNFR5. However, Ljlys11 single mutants and Ljlys11-Ljnfr5 double mutants are colonized by AMF similarly to WT plants (Rasmussen et al., 2016).

In RNS, there is strong host specificity that is known to depend at least in part on LCO structure. Indeed, Rhizobia strains usually produce major LCO structures with particular decorations. These decorations distinguish them from each other. Members of the phylogenetic group LYRIA from legumes are thus expected to have selectivity for LCO structure. This hypothesis is supported by genetic studies consisting in heterologous expression of orthologous genes from plant species interacting with Rhizobia producing different LCO structures (Radutoiu et al., 2007; Bensmihen et al., 2011). In contrast, there is no strict host specificity for AMS, suggesting that legume members of the phylogenetic group LYRIA might have acquired the ability to discriminate LCO decorations while ancestor proteins involved in the AMS did not display this property. However, whether LjNFR5 that binds Nod-factor has selectivity for LCO structure has not been demonstrated yet.

Altogether, current data indicate that genes belonging to the phylogenetic group LYRIA are involved in root endosymbioses. This is coherent with their absence in Brassicaceae that do not establish RNS nor AMS. However, only partial or no deficiency of the AMS establishment was observed in plants with knock-down or knock-out for genes from the subgroup LYRIA in tomato, P. andersonii, rice and L. japonicus (Op den Camp et al., 2011; Buendia et al., 2016; Miyata et al., 2016; Rasmussen et al., 2016) suggesting a redundancy for activation of the CSSP (which is required for AMS establishment) possibly through perception of signals other than LCOs.

\section{Subgroup LYRIB}

All analyzed plant species have one gene in the subgroup LYRIB except in the Brassicaceae. In contrast to most LYRs, members of the phylogenetic group LYRIB have a unique intron. We found that in L. japonicus, the exons are split in two different

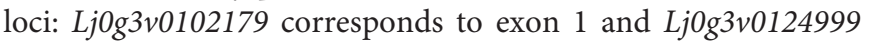
corresponds to exon 2. For this reason the lotus gene was not included in the phylogenetic analysis. Although to date, no biological role and has been reported for members of this phylogenetic group, phylogenetic proximity to subgroup LYRIA and absence of member in A. thaliana and B. rapa make the members of the phylogenetic group LYRIB good candidates to play a role in AMS establishment in higher plants.

\section{LysM-RLK LYRII (LYK Clade IV in Zhang et al., 2009)}

This phylogenetic group was found only in dicotyledons and is divided into two subgroups here called A and B, each containing one member in the species analyzed except the group LYRIIB 
in which there are no members in the Brassicaceae (Figure 4, Table 2, and Supplementary Figure S2). In contrast with the other phylogenetic groups, the number and even the position of introns vary between orthologs.

\section{LysM-RLK LYRIII (LYK Clades II and III in Zhang et al., 2009)}

In the phylogenetic group LYRIII, several gene duplications occurred. On the basis of the phylogenetic analysis, we divided this phylogenetic group into three subgroups (Figure 4, Table 2 and Supplementary Figure S2). However, these three subgroups were only detectable when the phylogeny was performed without using the Gblock tool (an algorithm for curing the alignment and that restricts the phylogeny analysis to conserved regions). In fact, when Gblock was integrated in the analysis, the phylogenetic group LYRIII was divided between monocotyledonous and the dicotyledonous group members (Supplementary Figure S4). It is important to note that Gblock had no effect on the organization of the other phylogenetic groups (data not shown). This suggests that phylogeny of subgroups in the phylogenetic group LYRIII is not robust.

\section{Subgroup LYRIIIA}

All the species analyzed here possess members of the phylogenetic subgroup LYRIIIA. Members of this group AtLYK4 (At2g23770) and LjLYS12 ( $L j 2 g 3 v 1828320$ ) were found to play a role in defense against pathogens. However, the biochemical characterization of MtLYR3 (Medtr5g019050) and LjLYS12 showed that these proteins are LCO binding proteins making difficult to understand the mechanisms in which they are involved.

In A. thaliana, responses to long-chain $\mathrm{CO}$ ( $\mathrm{CO} 6$ and $\mathrm{CO} 8)$ were decreased in Atlyk4 knock-out mutants but not totally abolished, suggesting that AtLYK4 plays a role in long-chain CO perception (Wan et al., 2012). AtLYK4 was also shown to play a positive role in defense against the fungal pathogen Alternaria brassicicola and the bacterial pathogen $P$. syringae (Wan et al., 2012). AtLYK4 was pulled down from A. thaliana solubilized membrane fractions using chitin beads and detected by mass spectrometry (Petutschnig et al., 2010; Wan et al., 2012). Affinity and selectivity of AtLYK4 for COs is unknown. Because the responses to long-chain COs were not abolished in Atlyk4 mutants, the authors suggested that an additional protein plays a redundant role in long-chain $\mathrm{CO}$ perception. This protein might be AtLYK5 described below. However, the implication of AtLYK4 in resistance to a bacterial pathogen questions the possible function of AtLYK4 as a CO binding protein and suggests a more general role in MAMP perception.

In L. japonicus, LjLYS12 (Lj2g3v1828320) expression is induced during infection by the oomycete Phytophthora palmivora and plant knock-outs for LjLYS12 are more susceptible to $P$. palmivora while no difference in RNS and AMS was detected compared to WT plants (Fuechtbauer et al., 2018). In M. truncatula, the biological role of MtLYR3 (Medtr5g019050) still remains unknown, however, Fliegmann et al. (2013) demonstrated that MtLYR3 has a high affinity for LCOs (Kd around $25 \mathrm{nM}$ ) reminiscent of a binding site characterized in a Medicago varia cell culture (Gressent et al., 1999). The protein was expressed in N. benthamiana leaves and LCO binding assays were performed on membrane fractions by competition between radiolabeled LCOs at a fixed concentration and ranges of concentrations of various unlabeled LCOs or COs. The MtLYR3 LCO binding site is specific for LCOs versus COs, however, it does not discriminate LCO decorations on the GlcNAc backbone. MtLYR3 orthologs in other legumes including LjLYS12, display similar affinities for LCOs as MtLYR3 except those of two Lupinus species incapable of forming the AMS, that do not bind LCOs (Malkov et al., 2016). This suggests that these proteins could play a role in the AMS at least in legume plants.

Interestingly, genes from the phylogenetic groups LYRIA and LYRIIIA are located at the same locus, as neighboring genes in opposite orientations in most dicotyledons (Figure 5). LCO binding properties of the proteins from these two phylogenetic subgroups are likely the consequence of a tandem duplication of an ancestral gene encoding a LCO binding protein.

In conclusion, members of the phylogenetic subgroup LYRIIIA appear to be involved in defense mechanisms while at least in legumes they can bind LCOs with high affinity. Additional studies are required to understand whether these genes could be involved in crosstalk between LCO perception and defense regulation.

\section{Subgroup LYRIIIB}

There is at least one member of the phylogenetic subgroup LYRIIIB in the genome of all the analyzed dicotyledons, except in the Brassicaceae. These genes are located next to the genes from the phylogenetic groups LYRIIIA in most dicotyledons except in legumes (Figure 5).

\section{Subgroup LYRIIIC}

Members of the phylogenetic subgroup LYRIIIC were not found in all the species analyzed here (they are absent in peach and tomato). AtLYK5 (At2g33580) and MtLYR4 (Medtr5g085790) are the best-characterized members of this phylogenetic group. They are involved in the perception of long-chain COs and resistance against fungal pathogens. An Atlyk5 knock-out mutant is strongly, although not fully, inhibited in responses to long-chain COs (CO6 to CO8) elicitation and is more susceptible to the fungus A. brassicicola (Cao et al., 2014). In contrast, the double mutant Atlyk4/Atlyk5 has completely abolished responses to long-chain COs but has a susceptibility to A. brassicicola similar to the Atlyk5 knock-out mutant (Cao et al., 2014). Affinity of AtLYK5 for COs was measured by ITC on AtLYK5 ECR produced in E. coli. An affinity of $1.72 \mu \mathrm{M}$ was found for $\mathrm{CO} 8$ while no binding to $\mathrm{CO} 4$ was detected. Using mutated versions of AtLYK5, it was shown that its CO binding activity is essential for its biological role. Key residues in the AtLYK5 CO binding site were identified by comparison with Ecp6, a fungal secreted protein containing 3 LysMs, which binds CO8 with high affinity (see below). Mutation in AtLYK5 Y128 and S206 led to inability of the tagged protein produced in planta to bind chitin beads and to the corresponding coding sequence to restore the ROS production in response to chitin when used to complement Atlyk5 plants (Cao et al., 2014). 
Two members of the phylogenetic subgroup LYRIIIC are found in legumes. In $M$. truncatula the MtLYR4 (Medtr5g085790) and MtLYR7 (Medtr3g080170) are found on different chromosomes while in L. japonicus LjLYS13 ( Lj2g3v2899910) and LjLYS14 (Lj2g3v2899900) are closely related genes, suggesting a more recent duplication event in L. japonicus. Mtlyr4 mutants showed increased susceptibility to the fungal pathogen B. cinerea (Bozsoki et al., 2017) and a loss of ROS production induced by $\mathrm{CO} 4$ or CO8. Furthermore, Mtlyr4 mutants showed a decreased MAPK 3/6 phosphorylation (a hallmark of MTI signaling) compared to WT when treated with $1 \mu \mathrm{M}$ CO8. LjLYS13 is expressed particularly in roots and up-regulated by CO8 treatment (Lohmann et al., 2010), suggesting a role in CO perception. LjLYS14 (Lj2g3v2899900) is expressed more ubiquitously and is also slightly induced by CO8. LjLYS13 and LjLYS14 expression is up-regulated in roots in presence of Rhizobia but not detected in nodules (Lohmann et al., 2010). In contrast to Mtlyr4 mutants, ROS production was similarly induced by CO4 or CO 8 in Ljlys 13 or Ljlys14 mutants as in WT plants (Bozsoki et al., 2017). This could be due to redundant functions of LjLYS13 and LjLYS14. Since it unlikely to obtain a double mutant by crossing single mutants because of the close proximity of the genes, it would be very informative to obtain the double mutant, for example using the CRISPR-Cas9 technology. The difference in the responses of Mtlyr4, LjLys13, and Ljlys14 to CO4 or CO8, reinforces the hypothesis that duplication events in the phylogenetic subgroup LYRIIIC were independent in $M$. truncatula and L. japonicus. In addition, MtLYR4 phosphorylation status was found to be affected by LCO treatment in a MtNFP-independent manner (Rose et al., 2012). Although the effect of LCOs on MtLYR4 phosphorylation is CSSP-independent, the MtLYR4 phosphorylation status itself appears to be controlled by the CSSP (Rose et al., 2012). However, Mtlyr4 mutants (as Ljlys13 and Ljlys14 mutants) are not affected in the RNS (Bozsoki et al., 2017).

\section{LysM-RLK LYRIV (Not Named in Zhang et al., 2009)}

Among the species analyzed, the phylogenetic group LYRIV contains members only in legume species and in peach (Figure 4, Table 2, and Supplementary Figure S2), suggesting that this group emerged in a common ancestor to these closely related plant species. Two members are found in M. truncatula and were reported in L. japonicus (Lohmann et al., 2010). Their kinase domains are very different from the other LYRs and closely related to RLKs from the wall associated kinase (WAK) subfamily (Arrighi et al., 2006).

\section{LysM-RLK LYKI (LYK Clade VI in Zhang et al., 2009)}

In the phylogenetic group LYKI the number of genes is highly variable between species (Figure 6, Table 3, and Supplementary Figure S3). Legumes display the highest number and diversity of members in this phylogenetic group ( 9 in M. truncatula and 5 in L. japonicus) whereas we only found 1 member in Brassicaceae and in B. distachyon. Members of this phylogenetic group are involved in the perception of the various GlcNAc-containing ligands (at least $\mathrm{CO}$ and $\mathrm{PGN}$ ) and have dual roles in endosymbiosis and defense. They might be co-receptors rather than ligand-binding proteins.

This phylogenetic group contains AtLYK1/AtCERK1 (At3g21630) that has been widely studied and first shown to be required for chitin responses. CO8-induced responses such as ROS production and MAP kinase phosphorylation are completely impaired in Atcerk1 knock-out mutants (Miya et al., 2007; Wan et al., 2008).

Contrasted results have been obtained concerning the affinity of AtCERK1 for chitin and COs. High affinity for chitin (Kd of $2 \mathrm{nM}$ ) has been reported (Iizasa et al., 2009) for the full length protein fused to GFP, produced in yeast, solubilized and purified to measure binding on chitin beads using a range of protein concentration. However, in the same study competition assays on the chitin beads using CO5, CO6, or CO8 led to half-maximal inhibitory concentration (IC50) of about $100 \mu \mathrm{M}$. Similarly, other studies showed a low affinity binding ( $K d$ of 44 and $455 \mu \mathrm{M}$ for CO8) using ITC with purified AtCERK1 ECR produced in insect cells and in E. coli, respectively (Liu T. et al., 2012; Cao et al., 2014). The huge differences between affinity reported for chitin and COs could be due to the methods used for affinity determination (quantification of AtCERK1:GFP fluorescence bound to chitin beads and ITC) or to differences in affinity for various degrees of GlcNAc polymerization (chitin vs. CO8).

Although AtCERK1 has been mainly studied for its role in chitin perception, it was shown that a Atcerk1 knock-out line is more sensitive to the pathogenic bacterium $P$. syringae (Wan et al., 2012) suggesting that AtCERK1 is also involved in perception of bacterial MAMPs. Indeed, AtCERK1 has been shown to be involved in PGN perception (Willmann et al., 2011) although it does not appear to directly bind PGN (Petutschnig et al., 2010; Willmann et al., 2011).

Similarly, tomato lines with reduced expression of SlLYK1/Bti9 (Solyc07g049180) and SlLYK3 (Solyc01g098410) were more susceptible to $P$. syringae (Zeng et al., 2012).

Finally, AtCERK1 was recently shown to be involved in perception of $\beta-1,3$ glucan hexasaccharides, which is not a GlcNAc-containing molecules (Mélida et al., 2018). Consistent with this observation, the Atcerk1 mutant was more susceptible to the oomycete Hyaloperonospora arabidopsidis, the cell wall of which is devoid of chitin (Mélida et al., 2018).

OsCERK1 (Os08g42580) is also involved in chitin and PGN signaling (Shimizu et al., 2010; Ao et al., 2014). Similarly to what has been observed for the Atcerk 1 mutant, Oscerk 1 mutants display strongly reduced responses to chitin, soluble CO7-8 or PGN treatment (ROS production, apoplastic alkalinization, genes regulation and callose deposition). As expected for a chitin-perception defective mutant, Oscerk1 mutant is more susceptible to the fungal pathogen M. oryzae (Kouzai et al., 2014a). However, unlike AtCERK1, OsCERK1 did not show any binding to insoluble colloidal chitin (Shinya et al., 2012). This difference of the biochemical properties between AtCERK1 and OsCERK1 was supported by the fact that OsCERK1 could not complement Atcerk1 for CO8-induced ROS responses (Shinya et al., 2012). Chimera consisting in AtCERK1 ECR and OsCERK1 ICR was able to partially rescue ROS production, suggesting that 
TABLE 3 | LysM-RLKs belonging to the phylogenetic groups LYKI, LYKII or LYKIII found in the 8 species analyzed.

\begin{tabular}{|c|c|c|c|c|c|c|c|c|}
\hline & M. truncatula & L. japonicus & P. persica & A. thaliana & B. rapa & S. lycopersicum & B. distachyon & O. sativa \\
\hline \multirow[t]{23}{*}{ LYKI } & Medtr5g086540 & Lj2g3v2904640 & & & & & & \\
\hline & LYK1 & LjLYS2 & & & & & & \\
\hline & Medtr5g086120 & & & & & & & \\
\hline & LYK4 & & & & & & & \\
\hline & Medtr5g086090 & & & & & & & \\
\hline & LYK5 & & & & & & & \\
\hline & Medtr5g086040 & Lj2g3v2904610 & & & & & & \\
\hline & LYK6 & LjLYS1 & & & & & & \\
\hline & Medtr5g086030 & & & & & & & \\
\hline & LYK7 & & & & & & & \\
\hline & Medtr5g086310 & & & & & & & \\
\hline & Medtr5g086330 & & & & & & & \\
\hline & LYK2 & & & & & & & \\
\hline & Medtr5g086130 & Lj2g3v2904690 & & & & Solyc01g098410 & & \\
\hline & LYK3 & LjNFR1 & & & & SILYK13 & & \\
\hline & Medtr3g080050 & Lj6g3v1055580 & Prupe.3G213100 & & & Solyc07g049180 & & \\
\hline & LYK9 & LjLYS6 & PpLYK2 & & & SILYK1/SIBti9 & & \\
\hline & Medtr2g024290 & Lj6g3v1812110 & Prupe.4G016100 & & & Solyc02g081050 & Bradi3g41590 & Os08g42580 \\
\hline & LYK8 & LjLYS7 & PpLYK1 & & & SILYK12 & BdLYK1 & OsCERK1 \\
\hline & & & & & & Solyc02g081040 & & Os09g33630 \\
\hline & & & & & & SILYK11 & & \\
\hline & & & & At3g21630 & Brara.E02055 & & & \\
\hline & & & & AtLYK1/AtCERK1 & & & & \\
\hline \multirow[t]{4}{*}{ LYKII } & Medtr5g033490 & Lj2g3v1415410 & Prupe.5G168000 & & & Solyc06g069610 & Bradi2g40627 & Os01G36550 \\
\hline & LYK10 & LjLYS3/EPR3 & PpLYK3 & & & SILYK14 & BdLYK2 & OsLYK1 \\
\hline & & & Prupe.1G247900 & & & & & \\
\hline & & & PpLYK4 & & & & & \\
\hline \multirow[t]{4}{*}{ LYKIII } & Medtr8g014500 & Lj3g3v2318170 & Prupe.3G058700 & At1g51940 & Brara.H00193 & Solyc03g121050 & Bradi2g49400 & Os01G53840 \\
\hline & LYK11 & LjLYS5 & PpLYK5 & AtLYK3 & & SILYK3 & BdLYK3 & \\
\hline & & Lj3g3v0290100 & & & Brara.F00223 & & & \\
\hline & & LjLYS4 & & & & & & \\
\hline
\end{tabular}

AtCERK1 binding properties are necessary for ROS responses in Arabidopsis.

Interestingly, an Oscerk1 knock-out line displayed a mycorrhizal phenotype (Miyata et al., 2014) with no root colonization at 15 days post inoculation (dpi), demonstrating a role of OsCERK1 in early fungal colonization. Some penetration sites and arbuscules were observed at 30 dpi. Similarly, rice plants with decreased level of OsCERK1 showed almost no AMF penetration at 6 weeks post inoculation (wpi; Zhang et al., 2015). This suggests that OsCERK1 is involved in perception of signals produced by AMF. Indeed, OsCERK1 is required for CO4 and CO5 perception as these molecules were unable to induce $\mathrm{Ca}^{2+}$ responses in Oscerk1 whereas Oscebip and Osnfr5 still display calcium spiking (Carotenuto et al., 2017).

Finally, OsCERK1, but not AtCERK1, was recently shown to be involved in perception of LPS, which is also not a GlcNAc-containing molecules (Desaki et al., 2018).

There is another member of the phylogenetic group LYKI in rice, $O s R L K 10$ (Os09g33630). It would be interesting to determine whether OsRLK10 is functionally redundant with OsCERK1 for one or both of the OsCERK1 functions.
Recently, Bozsoki et al. (2017) showed that members of the phylogenetic group LYKI in legumes, LjLYS6 (Lj6g3v1055580) and MtLYK9 (Medtr3g080050) are involved in defense. Ljlys6 and Mtlyr9 knock-out mutants are more susceptible than WT plants to the fungal pathogen $B$. cinerea. Moreover, responses to a range of COs (from CO4 to CO8) such as ROS production or MAPK3/6 phosphorylation were decreased compared to WT. To determine LjLYS6 affinity for COs, LjLYS6 ECR was produced in insect cells. After purification, it was deglycosylated and labeled with a fluorophore. Affinity for CO5 to CO8 was measured by MST using a range of CO concentrations. LjLYS6 was found to have a higher affinity for COs with long chains than with short chains, with a $K d$ of $38 \mu \mathrm{M}$ for CO8, $227 \mu \mathrm{M}$ for CO5 and no detectable binding of CO4. The affinity for CO8 is comparable to that found for AtCERK1 ECR produced in insect cells and measured by ITC (Liu T. et al., 2012). The crystal structure of LjLYS6 ECR (Bozsoki et al., 2017) was found to be similar to that of AtCERK1 ECR (Liu T. et al., 2012) although the authors could not observe LjLYS6 ECR bound to COs.

Other members of the phylogenetic group LYKI in legumes, LjNFR1 (Lj2g3v2904690) and MtLYK3 (Medtr5g086130) are 
involved in LCO (Nod-factor) perception in the RNS. The genes originate from duplication events specific to legumes (De Mita et al., 2014). Ljnfr1 mutants were impaired in nodulation and in the earliest responses to LCOs (Radutoiu et al., 2003). Apoplast alkalinization, that occurs immediately after LCO application and later responses such as root hair deformation (few hours after LCO application), were not observed in Ljnfr1 mutant lines. Whether LjNFR1 also plays a role in the AMS is a matter of debate. It has been shown that $\operatorname{Linfr} 1$ mutant lines display a lower colonization ratio compared to the WT 5 wpi (Zhang et al., 2015). Moreover, AMS marker genes and Myc-LCO-induced calcium spiking were reduced in an Ljnfrl mutant line compared to the WT (Zhang et al., 2015). In contrast, no difference in colonization ratio or fungal structure morphology was observed between a triple Ljnfr1-Ljnfr5-LjLys11 mutant line and the WT (Rasmussen et al., 2016). LjNFR1 LCO binding was analyzed with the same strategy as for LjNFR5 (phylogenetic group LYRIA). High affinity for LCO structure derivatives from $M$. loti main LCOs was found with a $K d$ of $4.9 \mathrm{nM}$ using SPR and with a $K d$ of $0.61 \mathrm{nM}$ using MST (Broghammer et al., 2012).

Structural differences were found between the LysM-RLKs of the phylogenetic group LYKI from species that establish endosymbiosis and those of the Brassicaceae which do establish endosymbioses. In all species except in the Brassicaceae, there is at least one member of the phylogenetic group LYKI that contains a specific motif in the kinase domain, YAQ in dicotyledons and YAR in monocotyledons, while AtCERK1 and Brara.E02055 have, respectively, one member that contains the residues TV or IV instead at this position (Figure 7). The YAQ/R motif has been demonstrated to be important for nodulation. Expression of a chimera containing the LjNFR1 ECR and the AtCERK1 ICR in a Ljnfr1 mutant was unable to restore nodulation (Nakagawa et al., 2011) in contrast to a LjNFR1-OsCERK1 chimera (Miyata et al., 2014). Replacement in AtCERK1 of the residues TV by YAQ led the chimera LjNFR1-AtCERK1 ${ }^{\mathrm{TV}-\mathrm{YAQ}}$ to restore nodulation in Ljnfr1. This suggests that the YAQ/R motif is associated with a symbiotic function either in the RNS as in LjNFR1 or in the AMS as in OsCERK1. However, in the dicotyledonous species analyzed that establish AMS, at least two paralogs bear the YAQ/R motifs and might have redundant roles (Figure 7). Since RNS is completely abolished in the Ljnfrl mutant, it is unlikely that another LysM-RLK has a redundant function in RNS. While it bears the YAQ/R motif, LjLYS6 was not found to be involved in the RNS nor in the AMS (Bozsoki et al., 2017). It could be hypothesized that LjLYS6 and LjLYS7 which both bear the YAQ/R motif have a redundant role in AMS establishment.

A knock-down of the LjLYS6 ortholog in pea, PsLYK9, conferred sensitivity to the fungal pathogen Fusarium culmorum. In addition, roots were also affected in transcriptomic responses to $\mathrm{CO} 5$, including the expression of genes that are upregulated

Medtr5g086540_(MtLYK1)
Medtr5g086120_(MtLYK4)
Medtr5g086090_(MtLYK5)
Medtr5g086040_(MtLYK5)
Medtr5g086030_(MtLYK6)
Medtr5g086310_(MtLYK2)
Medtr5g086130_(MtLYK3)
Medtr2g024290_(MtLYK8)
Medtr3g080050_(MtLYK9)
Lj2g3v2904640_(LjLYS2)
Lj2g3v2904610_(LjLYS1)
Lj2g3v2904690_(LjNFR1)
Lj6g3v1812110_(LjLYS7)
Lj6g3v1055580_(LjLYS6)
Prupe.4G016100
Prupe.3G213100
At3G21630_(AtCERK1)
Brara.E02055
Solyc02g081050_(SILYK12)
Solyc02g081040_(SILYK11)
Solyc07g049180_(SILYK1/SIBti9)
Solyc01g098410_(SILYK13)
Bradi3g41590_(BdLYK1)
Os08g42580_(OsLySM-RLK9/OsCERK1)
Os09g33630_(OsLySM-RLK10)

FIGURE 7 | Partial amino acid sequence alignment of members of the phylogenetic group LYKI. The YAQ/R motif present in the kinase domain is boxed in black. 
in WT roots colonized by AMF in pea suggesting that PsLYK9 is involved in symbiosis signaling (Leppyanen et al., 2017).

The LjNFR1 ortholog in M. truncatula, MtLYK3, has been demonstrated to be involved in nodulation. Mtlyk3 knock-down (Limpens et al., 2003) or missense (Wais et al., 2000; Catoira et al., 2001; Smit et al., 2007) lines were impaired in nodule formation and in Rhizobial colonization of M. truncatula roots but not in LCO responses such as apoplast alkalinization or calcium spiking. This led to the hypothesis that the genetic control of Rhizobial colonization is different between M. truncatula and L. japonicus and that MtLYK3 is involved LCO perception during Rhizobial colonization but not in LCO perception preceding colonization. This was consistent with the hypothesis of there are two receptors controlling responses to Nod-factors and rhizobial colonization in M. truncatula (Ardourel et al., 1994). However, a tandem duplication of MtLYK3 has occurred in M. truncatula. Two genes MtLYK3 and MtLYK2 (Medtr5g086310/Medtr5g086330) are LjNFR1 orthologs (Figure 6; De Mita et al., 2014). MtLYK2 might be redundant with MtLYK3. Actually, MtLYK2 contains as MtLYK3, the YAQ/R motif in its kinase domain. Although $M t L Y K 2$ is less expressed than MtLYK3 in roots (Limpens et al., 2003), it is possible that the MtLYK2 expression level in Mtlyk3 mutant lines is enough to ensure LCO responses preceding colonization but not Rhizobial colonization. This would explain the phenotypic difference between Mtlyk3 and Ljnfr1. Consistently, complementation experiments have shown that a chimeric protein containing LjNFR1 ECR and MtLYK3 ICR can restore the absence of nodulation in a Ljnfrl mutant (Nakagawa et al., 2011) suggesting a conservation of the function of these legume LysM-RLKs.

LjNFR1 and MtLYK3 are located in a cluster that contains 3 LYK genes in L. japonicus and 7 in M. truncatula. The number of LYK genes in this cluster is highly variable between legume species and could be partly responsible for host specificity through adaption to variation in the Nod-factor structure secreted by the Rhizobial symbionts as suggested by work in pea (Sulima et al., 2017).

Taken together, these data suggest evolution of members of the phylogenetic group LYKI for which the ancestral protein might have had a dual role in defense and AMS. Such a dual function is still found in rice. In this scenario, proteins have been subfunctionalized for a role in defense in Brassicaceae. In the other dicotyledonous species, the genes experienced several duplication events, which likely led to redundancy for a role in AMS and neofunctionalization for a role in RNS in legumes. Finally, because individual member of the phylogenetic group are involved in the perception of various molecules (at least PGN, long-chain COs and in $\beta$-1,3-glucan for AtCERK1, PGN, all COs and LPS for OsCERK1) leading to different biological responses and because they bear an active kinase in contrast to the LYRs, it is likely that these proteins are essential for signaling rather than for the specificity of ligand perception. This point will be discussed in the "Hetero-oligomeric complexes" section.

\section{LysM-RLK LYKII (Not Named in Zhang et al., 2009)}

In the Phylogenetic group LYKII, we found one ortholog in each species analyzed, except in the Brassicaceae (Figure 6,
Table 3, and Supplementary Figure S3). In the peach genome, gene duplication occurred and two copies are present. The only characterized member of the phylogenetic group LYKII is the L. japonicus member LjEPR3/LjLYS3 (Lj2g3v1415410) which has been shown to be implicated in the recognition of bacterial EPS and colonization by Rhizobia (Kawaharada et al., 2015, 2017). Knock-out or missense Ljepr3 mutants developed more nodules (although the number of nodules was extremely low) in the presence of a Rhizobial M. loti strain that produces an abnormal EPS structure $(e x o U)$ and which is almost unable to colonize WT L. japonicus (Kawaharada et al., 2015). In contrast to the M. loti exoU strain, a $M$. loti strain unable to produce EPS (exoB) was able to colonize WT L. japonicus, despite plants showed abnormal ITs (the cell invaginated structure that allow Rhizobia colonization in nodules) and intercellular Rhizobial colonization in nodules. Although to a lower extent, this phenotype was also observed in the Ljepr3 mutants inoculated with WT M. loti. The quantitative phenotypic differences between Ljepr 3 and WT plants suggest that EPR3 is, however, not the only actor in EPS perception (Kawaharada et al., 2017). LjEPR3 expression is induced by Nod-factors and by Rhizobia. In the presence of Rhizobia, its expression pattern in roots corresponds to the zone susceptible to Rhizobial colonization, around the ITs and in the nodule primordia. This suggests that $L j E P R 3$ is required all along the infection process (Kawaharada et al., 2017).

The orthologous gene in M. truncatula, MtLYK10 (Medtr5g033490) is also induced by Nod-factors and Rhizobia, and by Myc-factors and during the AMS (Mt gene atlas, Mtr.25148.1.S1_at; Camps et al., 2015). Similarly, orthologs in monocotyledons (Os01g36550 and Bradi2g40627) are induced during the AMS (Güimil et al., 2005, gene annotated OsAM191; and personal communication). The absence of any member of the phylogenetic group LYKII in Brassicaceae, together with the role of $L j E P R 3$ in Rhizobial colonization and the induction of the expression of various orthologs in the presence of root symbionts, suggest that the members of this phylogenetic group play a role in root endosymbioses. However, it has recently been reported that there is no ortholog of LjEPR3 in P. andersonii (van Velzen et al., 2018). LjEPR3 ECR was expressed in insect cells by using a baculovirus system. Binding to EPS was measured by biolayer interferometry using purified LjEPR3 ECR (Kawaharada et al., 2015) and a $K d$ of $2.7 \mu \mathrm{M}$ was found. Whether the recognition of EPS by LysM-RLKs is specific to L. japonicus or legumes remains unknown. It is therefore of interest to study a putative role in the AMS of non-legume members of the phylogenetic group LYKII and to determine their biochemical properties, especially their ability or not to bind bacterial EPS.

\section{LysM-RLK LYKIII (LYK Clade V in Zhang et al., 2009)}

In the phylogenetic group LYKIII, we identified at least one ortholog in each species, with duplications in B. rapa and in L. japonicus (Figure 6, Table 3, and Supplementary Figure S3). The only gene from this phylogenetic group that has been studied is AtLYK3 (At1g51940) and it was shown to act as a negative regulator of plant immunity in A. thaliana (Paparella et al., 2014). A T-DNA insertional mutant line displayed reduced symptoms in the presence of the fungal pathogen $B$. cinerea or the bacterial 
pathogen Pectobacterium carotovorum when compared to WT plants. In addition, basal expression in absence of pathogen of defense-related genes such as PAD3, a gene involved in phytoalexin biosynthesis, was higher in Atlyk3 mutants than in WT plants. AtLYK3 was also shown to be required for LCO perception in A. thaliana (Liang et al., 2013). The authors showed that treatment with LCO (at $100 \mathrm{nM}$ ) or CO4 (at $10 \mu \mathrm{M})$ partially inhibits (about 25\%) A. thaliana WT responses to the MAMPs flg22 or CO8. LCO effects on the attenuation of flg22 responses seem to occur through degradation of AtFLS2. LCO effects on flg22-induced ROS production were not observed in an Atlyk3 knock-out line and were stronger in an AtLYK3 overexpressing line suggesting that AtLYK3 is involved in LCO perception. Note that the data also suggest that in this Atlyk3 knock-out line, flg22-induced MAP kinase phosphorylation was reduced in absence of LCO.

\section{GENERAL DISCUSSION ON LySM-RLK ROLES AND FUNCTIONS}

\section{LysM-RLKs Function as Hetero-Oligomeric Complexes}

It is considered that RLKs function as hetero-oligomers composed of at least one protein that bind a signal molecule with high affinity through its ECR and one protein that transduce the signal through an active kinase domain in its ICR. The receptors for the MAMP peptides flg22 and elf18 occurs through high affinity binding to the LRR-RLKs AtFLS2 and AtEFR, respectively, and subsequent complex formation with the LRR-RLK AtBAK1 (Chinchilla et al., 2007; Schwessinger et al., 2011). AtFLS2 and AtEFR have non-RD kinases with weak activity (Schwessinger et al., 2011) compared to AtBAK1 which has a RD kinase and which is involved in multiple signaling pathways. AtFLS2 and AtEFR are then internalized following ligand perception in an AtBAK1-dependent manner (Mbengue et al., 2016).

A model for ligand perception by LysM-RLK/Ps proposes hetero-oligomers composed of at least one LYR/LYM and one LYK (Figure 8). It can be hypothesized that LYR or LYM proteins, lacking active kinase domain, are the partners that bind signal molecules with high affinity through their ECRs. High affinity likely corresponds to $K d$ values in the range of $\mathrm{nM}$ as measured for several LYRs and LYMs. Ligand binding to a LYR/LYM would induce (i) interaction with a LYK, which possesses an active kinase, or (ii) a change of conformation of the pre-existing LYR/LYM and LYK complex, leading to activation of the kinase of the LYK partner and signal transduction. Fitting this model, the LYMs OsCEBIP, OsLYP4, OsLYP6, AtLYM1, AtLYM2, AtLYM3, and MtLYM2 were found to bind PGN and/or COs and the LYRs AtLYK5, MtLYR3, LjLYS12, and LjNFR5 were shown to bind COs or LCOs. Except for OsLYP4 and OsLYP6, these proteins showed selectivity for a single type of ligand. Moreover, when their affinity was measured, these proteins were found to have high affinity for ligands. In contrast, the LYKs AtCERK1 and OsCERK1 were found to be involved in perception of multiple signals and to have low or no affinity for GlcNAc-containing

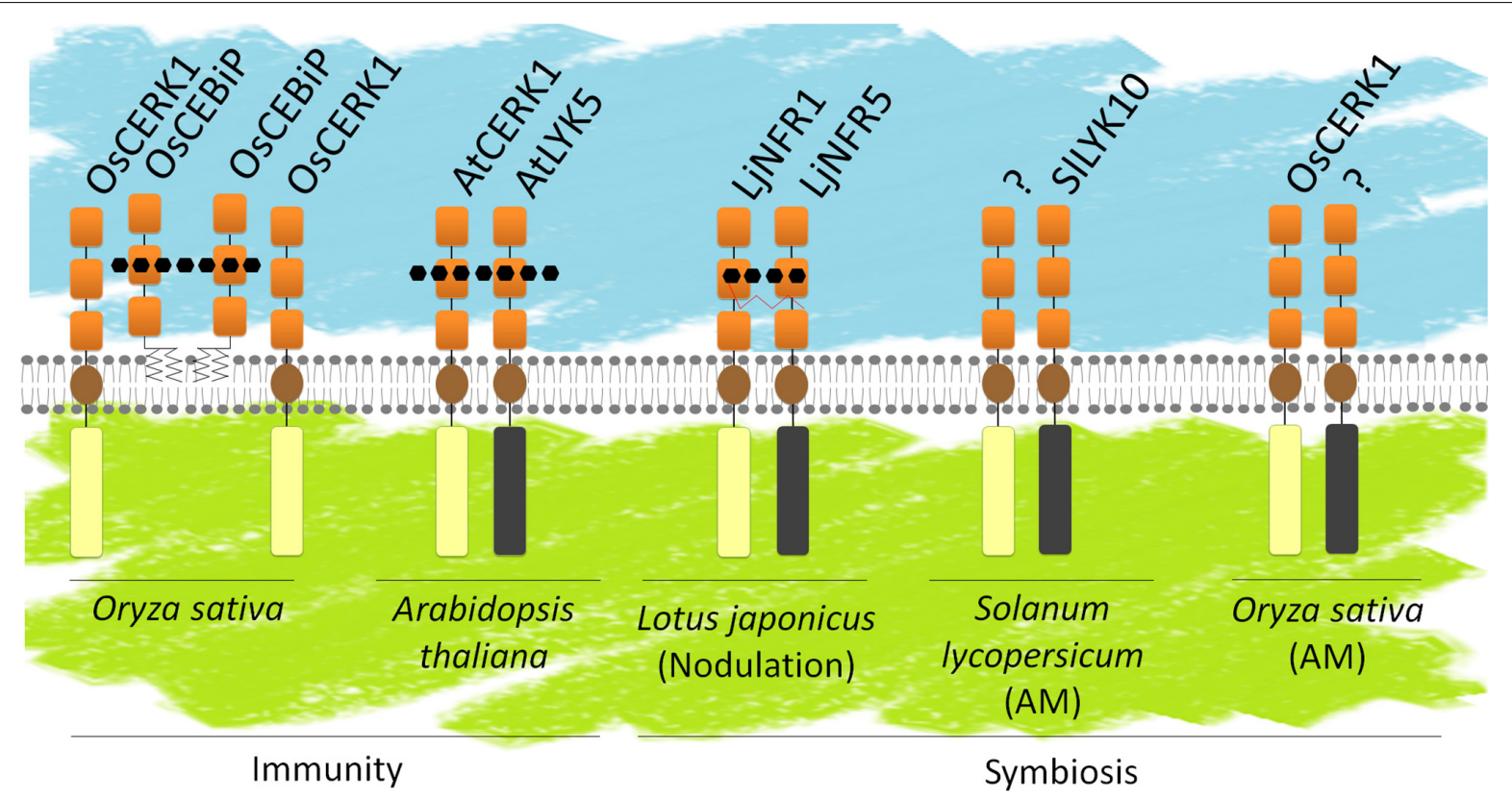

FIGURE 8 | LySM-RLK and/or LySM-RLP heterodimers. Known or hypothetical heterodimers involved in defense or symbiosis. Schematic representation of the LysM-RLKs/Ps as in Figure 1. LysM-RLKs with beige ICRs are LYKs (with active kinase domains), LysM-RLKs with gray ICRs are LYRs (with inactive kinase domains). Several models have been proposed in the literature: OsCEBIP might from a dimer that binds CO8 and interacts with OsCERK1 for signaling; AtLYK5 and AtCERK1 might both bind CO8; LjNFR5 and LjNFR1 might both bind LCOs. SILYK10 and OsCERK1 might interact with yet unidentified partners for perception of Myc-factors. 
ligands. Many genetic analyses actually suggest that LYMs/LYRs and LYKs interact, since mutants show similar phenotypes in responses to molecules or microorganisms (Table 4). Supporting the requirement of heterodimeric receptors to bind a ligand and transduce the signal, changes in host range during RNS or ligand specific responses were obtained by heterologously expressing couples of LYR/LYM and LYK proteins. Co-expression of LjNFR5 (LYRIA) and LjNFR1 (LYKI) in M. truncatula or in Lotus filicaulis modified host range (Radutoiu et al., 2007), while single proteins did not. Co-expression of chimeric LjNFR5-AtCERK1 and LjNFR1-AtCERK1 in A. thaliana led to production of ROS and expression of chitin-induced genes in response to LCO (Wang et al., 2014). Similarly, co-expression of chimeric OsCEBiP-LjNFR5 and OsCERK1-LjNFR1 in L. japonicus led to induction of LCO responsive genes in response to chitin and CO8 (Wang et al., 2014). Finally, physical interactions between LYMs/LYRs and LYKs have been demonstrated in planta. In rice cells, OsCEBIP, OsLYP4, and OsLYP6 (LYMII) interact with OsCERK1 (LYKI) in the presence of chitin (Shimizu et al., 2010; Ao et al., 2014). OsLYP4 and OsLYP6 also interact with OsCERK1 in presence of PGN (Ao et al., 2014). In A. thaliana, AtLYK5 (LYRII) interacts with AtCERK1 (LYKI), (Cao et al., 2014) under CO elicitation (CO6, CO7 and CO8 but not CO5). In M. truncatula physical interaction between MtNFP (LYRIA) and MtLYK3 (LYKI) was shown in nodules (Moling et al., 2014). It is unknown whether this interaction requires a ligand, although there is evidence for Nod-factor production by rhizobia inside legume nodules. Physical interaction of LjNFR5 (LYRIA) with LjNFR1 (LYKI) was shown in the absence of Nod-factors when expressed in a heterologous system (Madsen et al., 2011). However, it has to be noted that this was in a context of high expression levels. Physical interaction was also shown for MtLYR3 (LYRIIIA) and MtLYK3 (LYKI) in a heterologous system and in the absence of ligand and this interaction was decreased in the presence of LCO (Fliegmann et al., 2016).

After ligand perception, some LysM-RLKs might be internalized in endosomes for activation of signaling events taking place in endosomes and/or deactivation and degradation in the vacuole. This is the case for AtLYK5 internalization, which is induced by treatment with chitin. In contrast, AtCERK1 endocytosis does not seem to de induced by ligand perception. However, AtLYK5 phosphorylation and internalization depend on AtCERK1 kinase activity upon chitin treatment (Erwig et al., 2017).

Although most data on LysM-RLK/Ps fit with the model of a receptor complex consisting in a ligand binding protein and a protein involved in signaling, high affinity LCO binding of the kinase active LjNFR1 (LYKI) questions the model. Other high affinity ligand-binding proteins such as the LRR-RLK AtPERP1/2 which are receptors of endogenous peptides involved in wound signaling and innate immunity (Yamaguchi et al., 2006; Yamaguchi et al., 2010) and the LRR-RLK AtBRI1 which is the receptor of the Brassinosteroid hormone (He et al., 2000) have an active $\mathrm{RD}$ kinase. Recent data showing the involvement of OsCERK1 in perception of LPS and AtCERK1 in perception of $\beta$-1,3-glucan also open the possibility that LYKs participate to receptor complexes with other type of proteins than LysMRLK/Ps. Since LysM-RLKs are not expected to bind with high affinity to LPS, $\beta$-1,3-glucans or EPS and no LYR or LYM was found up to now to be involved in perception of these molecules, it is likely that AtCERK1, OsCERK1, and LjEPR3 interact with members of other protein families to form high affinity receptors for these molecules.

\section{Limitations in Ligand Binding Assays}

As reported above, the biochemical assays performed to characterize LysM-RLK/Ps have produced contrasting results. Differences in affinity for ligands of a LysM-RLK/P found between studies might be due to various reasons including the production system, the region of the protein used (e.g., full length versus ECR) and the binding assay. Because the E. coli system is not efficient for the formation of disulfide bridges that were shown to be essential for the function of several LysM-RLKs including MtNFP (Lefebvre et al., 2012) and LjEPR3/LjLYS3 (Kawaharada et al., 2015), the affinity measured with ECRs produced in E. coli might be underestimated. Indeed the AtCERK1 ECR produced in insect cells showed a $K d$ for CO8 of $44 \mu \mathrm{M}$ by ITC (Liu T. et al., 2012) while AtCERK1 ECR produced in $E$. coli showed a $K d$ for CO8 of $455 \mu \mathrm{M}$ (Cao et al., 2014) using the same binding assay. However, differences were

TABLE 4 | Known or hypothetical LysM-RLK/P heterodimers involved in defense or symbiosis.

\begin{tabular}{|c|c|c|c|c|}
\hline & $\begin{array}{c}\text { Genetic } \\
\text { interaction }\end{array}$ & Reference & $\begin{array}{l}\text { Physical } \\
\text { interaction }\end{array}$ & Reference \\
\hline AtLYM3/AtCERK1 & $x$ & Willmann et al., 2011 & & \\
\hline AtLYM1/AtCERK1 & $x$ & Willmann et al., 2011 & & \\
\hline AtLYK4/AtCERK1 & $x$ & Wan et al., 2012; Cao et al., 2014 & & \\
\hline AtLYK5/AtCERK1 & $x$ & Cao et al., 2014 & $x$ & Cao et al., 2014 \\
\hline OsLYP4/OsCERK1 & $x$ & Liu B. et al., 2012 & $x$ & Liu B. et al., 2012; Ao et al., 2014 \\
\hline OsLYP6/OsCERK1 & $x$ & Liu B. et al., 2012 & $x$ & Liu B. et al., 2012; Ao et al., 2014 \\
\hline OsCEBIP/OsCERK1 & $x$ & $\begin{array}{l}\text { Ao et al., 2014; Kouzai et al., 2014a; } \\
\text { Liu et al., } 2016\end{array}$ & $x$ & $\begin{array}{l}\text { Ao et al., 2014; Hayafune et al., } \\
\text { 2014; Liu et al., } 2016\end{array}$ \\
\hline MtNFP/MtLYK3 & $x$ & Limpens et al., 2003; Arrighi et al., 2006 & $x$ & Moling et al., 2014 \\
\hline LjNFR5/LJNFR1 & $x$ & $\begin{array}{l}\text { Madsen et al., 2003; Radutoiu et al., } \\
2003\end{array}$ & $x$ & Madsen et al., 2011 \\
\hline MtLYR3/MtLYK3 & & & $x$ & Fliegmann et al., 2016 \\
\hline
\end{tabular}


also found using various expression systems allowing formation of disulfide bridges. Expression of OsCEBIP full length protein in tobacco BY-2 cells suggested a $K d$ for CO8 around $100 \mathrm{nM}$, while using the OsCEBIP ECR produced in insect cells, the $\mathrm{Kd}$ for $\mathrm{CO} 8$ was about $4 \mu \mathrm{M}$. Differences affinity for ligands have also been found for the fungal effector Ecp6, a protein containing 3 LysMs which binds CO8. Using a recombinant Ecp6 purified from Pichia pastoris $K d$ between 3.7 and $4.5 \mu \mathrm{M}$ were found by ITC (de Jonge et al., 2010; Mentlak et al., 2012) while $K d s$ of $1.3 \mathrm{nM}$ or $380 \mathrm{~nm}$ were found by SPR using a CO8 immobilized or an effector-immobilized strategy, respectively. Using a recombinant Ecp6 purified from mammalian cells, a $K d$ of $280 \mathrm{pM}$ was found using ITC (Sánchez-Vallet et al., 2013). One reason explaining the differences between the results obtained with recombinant Ecp6 from yeast or mammalian cells might be the presence of yeast-derived COs co-purified with the protein that biased the analysis (Sánchez-Vallet et al., 2013). It is thus essential to compare affinities of proteins or domains produced similarly and characterized using the same binding assay.

Although half maximal incorporation in saturation experiments or half maximal inhibition in competition experiments correspond to $K d$ values in presence of a single binding site and appropriate receptor-ligand stoichiometry, proper $K d$ calculation requires either to know the ligand and the protein concentrations (for radiolabeled ligand assays, ITC, MST), or to measure the kinetics of association and dissociation (for SPR). With insoluble chitin, PGN or uncharacterized mixtures, it is impossible to determine the molecular concentration of the ligands and thus to determine the $K d$ from saturation or competition experiments. The $K d$ values found in the literature that are deduced from half maximal incorporation or half maximal inhibition experiments have thus to be considered with caution.

Another limitation to the current biochemical characterization and understanding of LysM-RLK function is the lack of studies on specificity of the protein-ligand interaction. Where controls have been used, they are often unrelated proteins and unrelated ligands. It is thus required to use other LysM-RLK/Ps and various structures of COs, LCOs or muropeptides as controls to be able to correlate binding properties and biological functions.

\section{Roles of LysM-RLKs in Plant Defense Vary Between Plant Species}

Plant LysM-RLK/Ps are involved in the perception of molecules that act as defense elicitors. Perception of chitin fragments relies on LYMs, LYRs and LYKs which may act in same or parallel pathways. AtLYK5 (LYRIIIC) was suggested to play a redundant role with AtLYK4 (LYRIIIA) for elicitation of defense responses through a signaling pathway depending on AtCERK1 [LYKI; (Cao et al., 2014)]. On the other hand, AtLYM2 (LYMII) was also shown to play a role in resistance to fungal pathogens. However, AtLYM2 is not required for the main ROS production in response to $\mathrm{CO} 8$ and has been proposed to play a role in plasmodesmata closure in response to COs. This occurs independently of AtCERK1 (Faulkner et al., 2013) raising the question of which co-receptor interacts with AtLYM2 for CO8 signaling. In rice, OsCEBIP, one of the AtLYM2 orthologs interacts with OsCERK1 (Shimizu et al., 2010) and is involved in the main ROS production in response to chitin fragments (Kaku et al., 2006). The differences found between Arabidopsis and rice for chitin perception point out the necessity of new studies on plant species in different phylogenetic clades to better understand how chitin perception has evolved in plants. Complementary roles of AtLYK5, AtLYK4, and AtLYM2 show that genetic screens based on measurement at the plant or organ level of classical responses to MAMP (such as $\mathrm{Ca}^{2+}$ flux, ROS production, marker gene induction) allow to identify genes responsible for strong responses (intense and/or produced in many cells). However, such screens do not permit to find genes controlling mechanisms in specific cells that could also play important roles in pathogen resistance. This should encourage the screening of mutant collections for a variety of responses to elicitors as well as to various pathogens.

\section{Pathogen Effectors Target or Compete LysM-RLKs to Avoid MTI Activation}

The importance of LysM-RLKs in plant defense is also highlighted by the fact that they are targeted by pathogen effectors. AtCERK1 is the target of AvrPtoB, a multi-domain and multi-function effector produced by several $P$. syringae pathovars (for review, Macho and Zipfel, 2015). AvrPtoB is known to contain an E3 ubiquitin ligase domain and to be injected in plant cells through the type III secretion system. AvrPtoB is able to ubiquitinate AtCERK1, inducing its degradation and suppressing MAMPS signaling (Gimenez-Ibanez et al., 2009). AvrPtoB is also able to interact with several AtCERK1 orthologs in tomato. Among them, SILYK1/SIBti9 displayed a reduced kinase activity in the presence of AvrPtoB (Zeng et al., 2012). Some fungal effectors such as Avr4 and Ecp6 from Cladosporium fulvum suppress plant immunity in another way, by competing with plant receptor for binding to chitin fragments or interfering with receptor dimerization (for review, Sánchez-Vallet et al., 2015). For instance, Mentlak et al. (2012) showed that Ecp6 from C. fulvum and Slp1 from M. oryzae are able to bind COs with high affinity. They appear to have higher affinity than OsCEBIP for CO8 (de Jonge et al., 2010; Mentlak et al., 2012; Sánchez-Vallet et al., 2013). When added exogenously, these effectors can compete for the binding of chitin fragments to OsCEBIP (de Jonge et al., 2010; Mentlak et al., 2012). Interestingly, these effectors are composed of three LysM as the LysM-RLK/Ps. However, they might originate from an independent association of LysMs, since the loops between the LysMs are different and the highly conserved CXC motifs found in LysM-RLK/Ps are not conserved between the LysM-RLK/Ps and the LysM effectors although disulfide bridges are also involved in packing together the LysM in the effectors. The association of three LysMs in different proteins for binding chitin fragments thus represents an example of convergent evolution. Fungal effectors with LysMs are found in many fungi (Bolton et al., 2008) including pathogens with different lifestyles or host ranges suggesting that the strategy of competing for chitin 
binding with plant receptors in order to avoid MTI activation is widespread in fungi. Several proteins containing LysM domains were shown to play a role in fungal pathogenicity: Mg3LysM in Mycosphaerella graminicola (Marshall et al., 2011), ChELP1 and ChELP2 in Colletotrichum higginsianum (Takahara et al., 2016) and Vd2LysM in Verticillium dahlia (Kombrink et al., 2017).

\section{Functions of LysM-RLKs Involved in RNS Vary Between Legume Species}

The importance of LysM-RLKs in the RNS has been unambiguously determined. Members of the phylogenetic group LYRIA and LYKI, act together for perception of Rhizobial Nod-factors. They are required for the earliest responses to LCOs, for Rhizobial colonization and for nodule development. Although demonstrated for L. japonicus LysM-RLKs, evidence for LCO binding to the M. truncatula LysM-RLKs involved in LCO perception are still lacking, questioning the similarity between these two legumes species for LCO perception. In addition, differences in sensitivity of root hairs for responses to LCOs have been detected between these species. Half of the root hairs showed calcium spiking in $M$. truncatula roots treated by up to $10^{-13} \mathrm{M}$ of Sinorhizobium meliloti Nod-factors (Oldroyd et al., 2001; Sun et al., 2015) while no root hairs showed calcium spiking in L. japonicus roots treated with $10^{-11} \mathrm{M}$ of $M$. loti Nod-factors (Sun et al., 2015). Most root hairs showed calcium spiking in L. japonicus roots treated with $10^{-9} \mathrm{M}$ of $\mathrm{M}$. loti Nod-factors (Oldroyd et al., 2001; Sun et al., 2015). Moreover, $S$. meliloti, a Rhizobial symbiont of $M$. truncatula, produces a LCO-IV(C16:2,S) as major Nod-factor (Lerouge et al., 1990) while $M$. loti, a Rhizobial symbiont of $L$. japonicus, produces LCO-V(C16:1,Cb,Fuc,Ac) as a major Nod-factor (Bek et al., 2010). A double unsaturation on the fatty acid and a sulfate groups are two LCO properties which are rare among the variety of Nod-factor structures produced by Rhizobia. Altogether, this suggests differences in LCO receptors between these two species. In contrast to $\mathrm{CO}$ binding proteins for which structures of their ECRs in interaction with COs have been resolved, no structural information is yet available for LCO binding proteins. Identification and characterization of the LCO binding site in LysM-RLKs will help to better understand the evolution of the LysM-RLK/P families.

\section{LysM-RLK/Ps Involved in Myc-Factor Perception Have Not Yet Been Identified}

The role of LysM-RLK/Ps and of Myc-factors in AMS remains unclear. LCOs and short-chain COs can activate the CSSP which is essential for AMS establishment, but it is unclear whether these signals have a redundant function. Until now, two orthologous LysM-RLKs, PaNFP, and SILYK10 have been shown to be involved in AMS establishment as AMF colonization is impaired in plants in which their expression is silenced (Op den Camp et al., 2011; Buendia et al., 2016). Because they are MtNFP/LjNFR5 orthologs, these proteins are expected to be LCO receptors although no data about their binding properties is yet published. Because these proteins are LYRs with inactive kinase, they might interact with yet unidentified LYK co-receptors.
Candidates are the members of the phylogenetic group LYKI bearing the YAQ/R motif.

Other LysM-RLK/Ps are thus expected to be short-chain CO receptors and could be involved in AMS. Evidence for shortchain CO perception was already published in the 1990s. A high affinity CO4/CO5 binding site was found in a tomato cell culture (Baureithel et al., 1994). Classical responses to MAMPs such as apoplast alkalinization were found in several Solanaceae (including tobacco and tomato) and in rice cell cultures (Felix et al., 1999). Interestingly, CO5 concentration required to induce such response in an Arabidopsis cell culture was much higher, suggesting there is no high affinity $\mathrm{CO} 4 / 5$ binding site in this plant species. Several years after, short-chain COs (CO4 and CO5) were shown to induce calcium spiking, which is currently considered a hallmark of symbiotic responses, in various plant species establishing AMS. Moreover abundance of $\mathrm{CO} 4$ and CO5 in AMF exudate is stimulated by strigolactones (Genre et al., 2013), a plant hormone which is known to promote AMF colonization. It was shown that the LysM-RLK OsCERK1 which is required for AMF colonization, is involved in CO4 perception (Carotenuto et al., 2017). OsCERK1 might interact with a highaffinity short-chain CO binding protein yet uncharacterized.

In conclusion, the role of LysM-RLKs in AMS has only started to be explored. No short-chain CO binding protein has been identified yet in any plant species. Only a few RLKs were found to be involved in AMS by the forward genetic screens or by reverse genetic approaches targeting single LysM-RLK/P performed up to now. This is likely due to the redundant functions of LysM-RLK/Ps for activation of the CSSP. Crosses to combine mutations in LysM-RLK/Ps or use of CRISPR-CAS9 technology targeting several LysM-RLK/Ps will be required to identify these genes. Considering the number of LysM-RLKs in legumes, such approaches have higher chance of success in non-legumes. However, differences between plant species in the mechanism of perception and/or in the responses to LCOs and COs as suggested by different roles of the orthologous genes SlLYK10/PaNFP and MtNFP/LjNFR5/OsNFR5 in AMS, make this research complicated. Determining the ability of LysM-RLK/Ps to bind to short-chain COs with high affinity and reverse genetics on combinations of LysM-RLKs in various plant species will help to better understand the importance of AMF symbiotic signals for establishment of the AMS.

\section{Various LysM-RLKs Have Dual Roles in Symbioses and Defense}

Symbiotic signals are structurally related to defense elicitors like chitin and PGN fragments. Consequently, it is logical to think that receptors should share similarities in terms of threedimensional structure and operating mode. Several questions arise from this statement. Under an evolutionary point of view, have symbiotic receptors evolved from MAMP receptors or vice versa? How can a plant deal with symbiotic partners that produce both symbiotic signals and MAMPs? In the complexity of the rhizosphere microbiome, how can plants distinguish and adapt their responses when surrounded by thousands of different microorganisms? Can pathogens use the symbiotic 
pathway to overcome plant defense? Even if most of these questions remain unanswered, some evidence suggests crosstalk between symbiosis and defense pathways. This might occur in part through dual functions of several LysM-RLKs in symbiosis and defense pathways as shown for OsCERK1 (essential for chitin/PGN signaling and for AMF colonization) and for MtNFP (essential for LCO signaling and involved in resistance to several pathogens).

Nod factors were found to transiently induce defense genes that are also induced by flg22 and chitin fragments (mix of $\mathrm{CO} 2$ to $\mathrm{CO} 8$ ), including $\mathrm{PR}$ proteins, peroxidases and transcription factors (El Yahyaoui et al., 2004; Nakagawa et al., 2011). Similarly, heterologous co-expression of two symbiotic LysM-RLKs, MtNFP and MtLYK3 or LjNFR5 and LjNFR1 induces defense responses and cell death, suggesting that these proteins can interact with signaling pathways involved in defense mechanisms (Madsen et al., 2011; Pietraszewska-Bogiel et al., 2013). Conversely, chitin fragments were found to transiently induce symbiotic genes independently of Nod-factor receptors (Nakagawa et al., 2011). Although the data were obtained through treatments with high concentration of the signal molecules and/or overexpression of the receptors, it shows possible crosstalk in the symbiosis and defense pathways.

Liang et al. (2013) demonstrated that in soybean and A. thaliana, perception of short-chain COs and LCOs interfere with responses induced by flg22. It is still undetermined whether the effect occurs by direct regulation of MAMP perception or signaling or because of a competition/desensitization of common actors involved in CO/LCO and MAMP signaling pathways. On the one hand, the observation of LCO effects in A. thaliana was surprising since this plant is not able to establish the AMS and has lost LYRI group members, known to be involved in LCO perception. On the other hand, the effect of LCO on flg22-induced responses in soybean was independent of the Nod-factor receptors suggesting that other LysM-RLK/Ps are involved in this mechanism.

An explanation to a crosstalk between symbiosis and defense pathway is that plants perceive MAMPs produced by their symbiotic partners and need to turn-off their defenses, at least locally for symbiosis establishment. Thus, it is expected that in plants able to establish the RNS and/or the AMS,

\section{REFERENCES}

Anisimova, M., and Gascuel, O. (2006). Approximate likelihood-ratio test for branches: a fast, accurate, and powerful alternative. Syst. Biol. 55, 539-552. doi: 10.1080/10635150600755453

Antolín-Llovera, M., Ried, M. K., Binder, A., and Parniske, M. (2012). Receptor kinase signaling pathways in plant-microbe interactions. Annu. Rev. Phytopathol. 50, 451-473. doi: 10.1146/annurev-phyto-081211-173002

Ao, Y., Li, Z., Feng, D., Xiong, F., Liu, J., Li, J. F., et al. (2014). OsCERK1 and OsRLCK176 play important roles in peptidoglycan and chitin signaling in rice innate immunity. Plant J. 80, 1072-1084. doi: 10.1111/tpj. 12710

Ardourel, M., Demont, N., Debelle, F. D., Maillet, F., Debilly, F., Prome, J. C., et al. (1994). Rhizobium meliloti lipooligosaccharide nodulation factors - different structural requirements for bacterial entry into target root hair-cells and induction of plant symbiotic developmental responses. Plant Cell 6, 1357-1374. doi: $10.1105 /$ tpc.6.10.1357 symbiotic signals have a direct effect on defense mechanisms by down-regulating them. The mechanism involved in such crosstalk is, however, unknown. Up to now, the signaling pathways activated by MAMPs and the symbiotic signals appear to be different suggesting that the crosstalk between defense and symbioses occurs downstream. To better understand the role of LysM-RLK/Ps in this crosstalk, studies similar to that performed by Liang et al. (2013) have to be performed in lines mutated in LysM-RLK/Ps from species that establish the RNS and/or the AMS. Moreover, local and systemic effects of symbiotic signals on defense should be analyzed.

\section{AUTHOR CONTRIBUTIONS}

LB, AG, TW, and BL wrote the text. LC constructed the phylogeny trees.

\section{FUNDING}

This work was supported by the ANR "WHEATSYM" (ANR16-CE20-0025-01) and the "Laboratoire d'Excellence (LABEX)" TULIP (ANR-10-LABX-41). AG's fellowship was funded by Région Occitanie and INRA Department of Plant Health and Environment (SPE). TW's fellowship was funded by the Chinese Scholarship Council (CSC). LB's fellowship was funded by French "Ministère de l’Enseignement Supérieur et de la Recherche".

\section{ACKNOWLEDGMENTS}

We are grateful to Clare Gough and Julie Cullimore for critical reading of the manuscript.

\section{SUPPLEMENTARY MATERIAL}

The Supplementary Material for this article can be found online at: https://www.frontiersin.org/articles/10.3389/fpls.2018.01531/ full\#supplementary-material

Arrighi, J. F., Barre, A., Ben Amor, B., Bersoult, A., Soriano, L. C., Mirabella, R., et al. (2006). The Medicago truncatula lysin motif-receptor-like kinase gene family includes NFP and new nodule-expressed genes. Plant Physiol. 142, 265-279. doi: 10.1104/pp.106.084657

Bateman, A., and Bycroft, M. (2000). The structure of a LysM domain from E. coli membrane-bound lytic murein transglycosylase D (MltD). J. Mol. Biol. 299, 1113-1119. doi: 10.1006/jmbi.2000.3778

Baureithel, K., Felix, G., and Boller, T. (1994). Specific, high affinity binding of chitin fragments to tomato cells and membranes. Competitive inhibition of binding by derivatives of chitooligosaccharides and a Nod factor of Rhizobium. J. Biol. Chem. 269, 17931-17938.

Beck, M., Heard, W., Mbengue, M., and Robatzek, S. (2012). The INs and OUTs of pattern recognition receptors at the cell surface. Curr. Opin. Plant Biol. 15, 367-374. doi: 10.1016/j.pbi.2012.05.004

Bek, A. S., Sauer, J., Thygesen, M. B., Duus, J., Petersen, B. O., Thirup, S., et al. (2010). Improved characterization of nod factors and genetically based variation in LysM Receptor domains identify amino acids expendable for nod factor 
recognition in Lotus spp. Mol. Plant Microbe Interact. 23, 58-66. doi: 10.1094/ MPMI- 23-1-0058

Belkhadir, Y., Yang, L., Hetzel, J., Dangl, J. L., and Chory, J. (2014). The growthdefense pivot: crisis management in plants mediated by LRR-RK surface receptors. Trends Biochem. Sci. 39, 447-456. doi: 10.1016/j.tibs.2014.06.006

Ben, C., Toueni, M., Montanari, S., Tardin, M. C., Fervel, M., Negahi, A., et al. (2013). Natural diversity in the model legume Medicago truncatula allows identifying distinct genetic mechanisms conferring partial resistance to Verticillium wilt. J. Exp. Bot. 64, 317-332. doi: 10.1093/jxb/ers337

Ben Amor, B., Shaw, S. L., Oldroyd, G. E. D., Maillet, F., Penmetsa, R. V., Cook, D., et al. (2003). The NFP locus of Medicago truncatula controls an early step of Nod factor signal transduction upstream of a rapid calcium flux and root hair deformation. Plant J. 34, 495-506. doi: 10.1046/j.1365-313X.2003.01743.x

Bensmihen, S., de Billy, F., and Gough, C. (2011). Contribution of NFP LysM domains to the recognition of Nod factors during the Medicago truncatula/Sinorhizobium meliloti symbiosis. PLoS One 6:e26114. doi: 10.1371/ journal.pone.0026114

Bielnicki, J., Devedjiev, Y., Derewenda, U., Dauter, Z., Joachimiak, A., and Derewenda, Z. S. (2006). B. subtilis ykuD protein at 2.0 A resolution: insights into the structure and function of a novel, ubiquitous family of bacterial enzymes. Proteins 62, 144-151. doi: 10.1002/prot.20702

Bolton, M. D., van Esse, H. P., Vossen, J. H., de Jonge, R., Stergiopoulos, I., Stulemeijer, I. J., et al. (2008). The novel Cladosporium fulvum lysin motif effector Ecp6 is a virulence factor with orthologues in other fungal species. Mol. Microbiol. 69, 119-136. doi: 10.1111/j.1365-2958.2008.06270.x

Bono, J., Fliegmann, J., Gough, C., and Cullimore, J. (2018). "Expression and function of the Medicago truncatula lysin motif receptor-like kinase (LysMRLK) gene family in the legume-rhizobia symbiosis," in The Model Legume Medicago truncatula, ed. F. de Bruijn (Hoboken, NJ: John Wiley \& Sons Inc).

Bozsoki, Z., Cheng, J., Feng, F., Gysel, K., Vinther, M., Andersen, K. R., et al. (2017) Receptor-mediated chitin perception in legume roots is functionally separable from Nod factor perception. Proc. Natl. Acad. Sci. U.S.A. 114, 8118-8127. doi: $10.1073 /$ pnas.1706795114

Broghammer, A., Krusell, L., Blaise, M., Sauer, J., Sullivan, J. T., Maolanon, N., et al. (2012). Legume receptors perceive the rhizobial lipochitin oligosaccharide signal molecules by direct binding. Proc. Natl. Acad. Sci. U.S.A. 109, 13859-13864. doi: 10.1073/pnas.1205171109

Buendia, L., Wang, T. M., Girardin, A., and Lefebvre, B. (2016). The LysM receptorlike kinase SILYK10 regulates the arbuscular mycorrhizal symbiosis in tomato. New Phytol. 210, 184-195. doi: 10.1111/nph.13753

Buist, G., Steen, A., Kok, J., and Kuipers, O. P. (2008). LysM, a widely distributed protein motif for binding to (peptido)glycans. Mol. Microbiol. 68, 838-847. doi: 10.1111/j.1365-2958.2008.06211.x

Camps, C., Jardinaud, M. F., Rengel, D., Carrère, S., Hervé, C., Debellé, F., et al. (2015). Combined genetic and transcriptomic analysis reveals three major signalling pathways activated by Myc-LCOs in Medicago truncatula. New Phytol. 208, 224-240. doi: 10.1111/nph.13427

Cao, Y., Liang, Y., Tanaka, K., Nguyen, C. T., Jedrzejczak, R. P., Joachimiak, A., et al. (2014). The kinase LYK5 is a major chitin receptor in Arabidopsis and forms a chitin-induced complex with related kinase CERK1. eLife 3:e03766. doi: 10.7554/eLife.03766

Carotenuto, G., Chabaud, M., Miyata, K., Capozzi, M., Takeda, N., Kaku, H., et al. (2017). The rice LysM receptor-like kinase OsCERK1 is required for the perception of short-chain chitin oligomers in arbuscular mycorrhizal signaling. New Phytol. 214, 1440-1446. doi: 10.1111/nph.14539

Catoira, R., Timmers, A. C. J., Maillet, F., Galera, C., Penmetsa, R. V., Cook, D. et al. (2001). The HCL gene of Medicago truncatula controls Rhizobiuminduced root hair curling. Development 128, 1507-1518.

Chinchilla, D., Bauer, Z., Regenass, M., Boller, T., and Felix, G. (2006). The Arabidopsis receptor kinase FLS2 binds flg22 and determines the specificity of flagellin perception. Plant Cell 18, 465-476. doi: 10.1105/tpc.105.036574

Chinchilla, D., Zipfel, C., Robatzek, S., Kemmerling, B., Nürnberger, T., Jones, J. D., et al. (2007). A flagellin-induced complex of the receptor FLS2 and BAK1 initiates plant defence. Nature 448, 497-500. doi: 10.1038/nature05999

Combier, J. P., Küster, H., Journet, E. P., Hohnjec, N., Gamas, P., and Niebel, A. (2008). Evidence for the involvement in nodulation of the two small putative regulatory peptide-encoding genes MtRALFL1 and MtDVL1. Mol. Plant Microbe Interact. 21, 1118-1127. doi: 10.1094/MPMI-21-8-1118 de Jonge, R., van Esse, H. P., Kombrink, A., Shinya, T., Desaki, Y., Bours, R., et al. (2010). Conserved fungal LysM effector Ecp6 prevents chitin-triggered immunity in plants. Science 329, 953-955. doi: 10.1126/science.1190859

De Mita, S., Streng, A., Bisseling, T., and Geurts, R. (2014). Evolution of a symbiotic receptor through gene duplications in the legume-rhizobium mutualism. New Phytol. 201, 961-972. doi: 10.1111/nph.12549

Deakin, W. J., and Broughton, W. J. (2009). Symbiotic use of pathogenic strategies: rhizobial protein secretion systems. Nat. Rev. Microbiol. 7, 312-320. doi: 10. 1038/nrmicro2091

Delaux, P., Varala, K., Edger, P., Coruzzi, G., Pires, J., and Ane, J. (2014). Comparative phylogenomics uncovers the impact of symbiotic associations on host genome evolution. PLoS Genet. 10:e1004487. doi: 10.1371/journal.pgen. 1004487

Desaki, Y., Kouzai, Y., Ninomiya, Y., Iwase, R., Shimizu, Y., Seko, K., et al. (2018). OsCERK1 plays a crucial role in the lipopolysaccharide-induced immune response of rice. New Phytol. 217, 1042-1049. doi: 10.1111/nph.14941

El Yahyaoui, F., Küster, H., Ben Amor, B., Hohnjec, N., Pühler, A., Becker, A., et al. (2004). Expression profiling in Medicago truncatula identifies more than 750 genes differentially expressed during nodulation, including many potential regulators of the symbiotic program. Plant Physiol. 136, 3159-3176. doi: 10. 1104/pp.104.043612

Erwig, J., Ghareeb, H., Kopischke, M., Hacke, R., Matei, A., Petutschnig, E., et al. (2017). Chitin-induced and CHITIN ELICITOR RECEPTOR KINASE1 (CERK1) phosphorylation-dependent endocytosis of Arabidopsis thaliana LYSIN MOTIF-CONTAINING RECEPTOR-LIKE KINASE5 (LYK5). New Phytol. 215, 382-396. doi: 10.1111/nph.14592

Faulkner, C., Petutschnig, E., Benitez-Alfonso, Y., Beck, M., Robatzek, S., Lipka, V., et al. (2013). LYM2-dependent chitin perception limits molecular flux via plasmodesmata. Proc. Natl. Acad. Sci. U.S.A. 110, 9166-9170. doi: 10.1073/pnas. 1203458110

Felix, G., Duran, J. D., Volko, S., and Boller, T. (1999). Plants have a sensitive perception system for the most conserved domain of bacterial flagellin. Plant J. 18, 265-276. doi: 10.1046/j.1365-313X.1999.00265.x

Felle, H. H., Kondorosi, E., Kondorosi, A., and Schultze, M. (1996). Rapid alkalinization in alfalfa root hairs in response to rhizobial lipochitooligosaccharide signals. Plant J. 10, 295-301. doi: 10.1046/j.1365313X.1996.10020295.X

Fliegmann, J., and Bono, J. J. (2015). Lipo-chitooligosaccharidic nodulation factors and their perception by plant receptors. Glycoconj. J. 32, 455-464. doi: 10.1007/ s10719-015-9609-3

Fliegmann, J., Canova, S., Lachaud, C., Uhlenbroich, S., Gasciolli, V., Pichereaux, C., et al. (2013). Lipo-chitooligosaccharidic symbiotic signals are recognized by LysM receptor-like kinase LYR3 in the legume Medicago truncatula. ACS Chem. Biol. 8, 1900-1906. doi: 10.1021/cb400369u

Fliegmann, J., and Felix, G. (2016). Immunity: flagellin seen from all sides. Nat. Plants 2:16136. doi: 10.1038/nplants.2016.136

Fliegmann, J., Jauneau, A., Pichereaux, C., Rosenberg, C., Gasciolli, V., Timmers, A. C., et al. (2016). LYR3, a high-affinity LCO-binding protein of Medicago truncatula, interacts with LYK3, a key symbiotic receptor. FEBS Lett. 590, 1477-1487. doi: 10.1002/1873-3468.12191

Fliegmann, J., Uhlenbroich, S., Shinya, T., Martinez, Y., Lefebvre, B., Shibuya, N., et al. (2011). Biochemical and phylogenetic analysis of CEBiP-like LysM domain-containing extracellular proteins in higher plants. Plant Physiol. Biochem. 49, 709-720. doi: 10.1016/j.plaphy.2011.04.004

Fuechtbauer, W., Yunusov, T., Bozsóki, Z., Gavrin, A., James, E. K., Stougaard, J., et al. (2018). LYS12 LysM receptor decelerates Phytophthora palmivora disease progression in Lotus japonicus. Plant J. 93, 297-310. doi: 10.1111/tpj.13785

Genre, A., Chabaud, M., Balzergue, C., Puech-Pages, V., Novero, M., Rey, T., et al. (2013). Short-chain chitin oligomers from arbuscular mycorrhizal fungi trigger nuclear $\mathrm{Ca}_{2}{ }^{+}$spiking in Medicago truncatula roots and their production is enhanced by strigolactone. New Phytol. 198, 179-189. doi: 10.1111/nph.12146

Gimenez-Ibanez, S., Hann, D. R., Ntoukakis, V., Petutschnig, E., Lipka, V., and Rathjen, J. P. (2009). AvrPtoB targets the LysM receptor kinase CERK1 to promote bacterial virulence on plants. Curr. Biol. 19, 423-429. doi: 10.1016/j. cub.2009.01.054

Gomez, S. K., and Harrison, M. J. (2009). Laser microdissection and its application to analyze gene expression in arbuscular mycorrhizal symbiosis. Pest Manag. Sci. 65, 504-511. doi: 10.1002/ps.1715 
Gomez-Gomez, L., and Boller, T. (2000). FLS2: an LRR receptor-like kinase involved in the perception of the bacterial elicitor flagellin in Arabidopsis. Mol. Cell. 5, 1003-1011. doi: 10.1016/S1097-2765(00)80265-8

Gough, C., and Jacquet, C. (2013). Nod factor perception protein carries weight in biotic interactions. Trends Plant Sci. 18, 566-574. doi: 10.1016/j.tplants.2013. 06.001

Gressent, F., Drouillard, S., Mantegazza, N., Samain, E., Geremia, R. A., Canut, H., et al. (1999). Ligand specificity of a high-affinity binding site for lipochitooligosaccharidic Nod factors in Medicago cell suspension cultures. Proc. Natl. Acad. Sci. U.S.A. 96, 4704-4709. doi: 10.1073/pnas.96.8.4704

Güimil, S., Chang, H. S., Zhu, T., Sesma, A., Osbourn, A., Roux, C., et al. (2005). Comparative transcriptomics of rice reveals an ancient pattern of response to microbial colonization. Proc. Natl. Acad. Sci. U.S.A. 102, 8066-8070. doi: 10.1073/pnas.0502999102

Guindon, S., Dufayard, J. F., Lefort, V., Anisimova, M., Hordijk, W., and Gascuel, O. (2010). New algorithms and methods to estimate maximumlikelihood phylogenies: assessing the performance of PhyML 3.0. Syst. Biol. 59, 307-321. doi: 10.1093/sysbio/syq010

Gutjahr, C., and Parniske, M. (2013). Cell and developmental biology of arbuscular mycorrhiza symbiosis. Annu. Rev. Cell Dev. Biol. 29, 593-617. doi: 10.1146/ annurev-cellbio-101512-122413

Harris, J. M., Wais, R., and Long, S. R. (2003). Rhizobium-lnduced calcium spiking in Lotus japonicus. Mol. Plant Microbe Interact. 16, 335-341. doi: 10.1094/ MPMI.2003.16.4.335

Hayafune, M., Berisio, R., Marchetti, R., Silipo, A., Kayama, M., Desaki, Y., et al. (2014). Chitin-induced activation of immune signaling by the rice receptor CEBiP relies on a unique sandwich-type dimerization. Proc. Natl. Acad. Sci. U.S.A. 111, E404-E413. doi: 10.1073/pnas.1312099111

Hazak, O., and Hardtke, C. S. (2016). CLAVATA 1-type receptors in plant development. J. Exp. Bot. 67, 4827-4833. doi: 10.1093/jxb/erw247

He, Z., Wang, Z. Y., Li, J., Zhu, Q., Lamb, C., Ronald, P., et al. (2000). Perception of brassinosteroids by the extracellular domain of the receptor kinase BRI1. Science 288, 2360-2363. doi: 10.1126/science.288.5475.2360

Herrbach, V., Chirinos, X., Rengel, D., Agbevenou, K., Vincent, R., Pateyron, S., et al. (2017). Nod factors potentiate auxin signaling for transcriptional regulation and lateral root formation in Medicago truncatula. J. Exp. Bot. 68, 569-583. doi: 10.1093/jxb/erw474

Hohnjec, N., Czaja-Hasse, L. F., Hogekamp, C., and Küster, H. (2015). Pre-announcement of symbiotic guests: transcriptional reprogramming by mycorrhizal lipochitooligosaccharides shows a strict co-dependency on the GRAS transcription factors NSP1 and RAM1. BMC Genomics 16:994. doi: 10. 1186/s12864-015-2224-7

Iizasa, E., Mitsutomi, M., and Nagano, Y. (2009). Direct binding of a plant LysM receptor-like kinase, LysM RLK1/CERK1, to chitin in vitro. J. Biol. Chem. 285, 2996-3004. doi: 10.1074/jbc.M109.027540

Ito, Y., Kaku, H., and Shibuya, N. (1997). Identification of a high-affinity binding protein for $\mathrm{N}$-acetylchitooligosaccharide elicitor in the plasma membrane of suspension-cultured rice cells by affinity labeling. Plant J. 12, 347-356. doi: 10.1046/j.1365-313X.1997.12020347.x

Kaku, H., Nishizawa, Y., Ishii-Minami, N., Akimoto-Tomiyama, C., Dohmae, N., Takio, K., et al. (2006). Plant cells recognize chitin fragments for defense signaling through a plasma membrane receptor. Proc. Natl. Acad. Sci. U.S.A. 103, 11086-11091. doi: 10.1073/pnas.0508882103

Kamel, L., Tang, N., Malbreil, M., San Clemente, H., Le Marquer, M., Roux, C., et al. (2017). The comparison of expressed candidate secreted proteins from two arbuscular mycorrhizal fungi unravels common and specific molecular tools to invade different host plants. Front. Plant Sci. 8:124. doi: 10.3389/fpls.2017.00124

Katoh, K., Misawa, K., Kuma, K., and Miyata, T. (2002). MAFFT: a novel method for rapid multiple sequence alignment based on fast Fourier transform. Nucleic Acids Res. 30, 3059-3066. doi: 10.1093/nar/gkf436

Kawaharada, Y., Kelly, S., Nielsen, M. W., Hjuler, C. T., Gysel, K., Muszyński, A., et al. (2015). Receptor-mediated exopolysaccharide perception controls bacterial infection. Nature 523, 308-312. doi: 10.1038/nature14611

Kawaharada, Y., Nielsen, M. W., Kelly, S., James, E. K., Andersen, K. R., Rasmussen, S. R., et al. (2017). Differential regulation of the Epr3 receptor coordinates membrane-restricted rhizobial colonization of root nodule primordia. Nat. Commun. 8:14534. doi: 10.1038/ncomms14534
Kishimoto, K., Kouzai, Y., Kaku, H., Shibuya, N., Minami, E., and Nishizawa, Y. (2010). Perception of the chitin oligosaccharides contributes to disease resistance to blast fungus Magnaporthe oryzae in rice. Plant J. 64, 343-354. doi: 10.1111/j.1365-313X.2010.04328.x

Klaus-Heisen, D., Nurisso, A., Pietraszewska-Bogiel, A., Mbengue, M., Camut, S., Timmers, T., et al. (2011). Structure-function similarities between a plant receptor-like kinase and the human interleukin-1 receptor-associated kinase- 4 . J. Biol. Chem. 286, 11202-11210. doi: 10.1074/jbc.M110.186171

Kloppholz, S., Kuhn, H., and Requena, N. (2011). A secreted fungal effector of Glomus intraradices promotes symbiotic biotrophy. Curr. Biol. 21, 1204-1209. doi: 10.1016/j.cub.2011.06.044

Koharudin, L. M., Debiec, K. T., and Gronenborn, A. M. (2015). Structural insight into fungal cell wall recognition by a CVNH protein with a single LysM domain. Structure 23, 2143-2154. doi: 10.1016/j.str.2015.07.023

Kombrink, A., Rovenich, H., Shi-Kunne, X., Rojas-Padilla, E., van den Berg, G. C., Domazakis, E., et al. (2017). Verticillium dahliae LysM effectors differentially contribute to virulence on plant hosts. Mol. Plant Pathol. 18, 596-608. doi: $10.1111 / \mathrm{mpp} .12520$

Kouzai, Y., Mochizuki, S., Nakajima, K., Desaki, Y., Hayafune, M., Miyazaki, H., et al. (2014a). Targeted gene disruption of OsCERK1 reveals its indispensable role in chitin perception and involvement in the peptidoglycan response and immunity in rice. Mol. Plant Microbe Interact. 27, 975-982. doi: 10.1094/ MPMI-03-14-0068-R

Kouzai, Y., Nakajima, K., Hayafune, M., Ozawa, K., Kaku, H., Shibuya, N., et al. (2014b). CEBiP is the major chitin oligomer-binding protein in rice and plays a main role in the perception of chitin oligomers. Plant Mol. Biol. 84, 519-528. doi: 10.1007/s11103-013-0149-6

Kuchitsu, K., Yazaki, Y., Sakano, K., and Shibuya, N. (1997). Transient cytoplasmic $\mathrm{pH}$ change and ion fluxes through the plasma membrane in suspensioncultured rice cells triggered by $\mathrm{N}$-acetylchitooligosaccharide elicitor. Plant Cell Physiol. 38, 1012-1018. doi: 10.1093/oxfordjournals.pcp.a029265

Lee, W. S., Rudd, J. J., Hammond-Kosack, K. E., and Kanyuka, K. (2014). Mycosphaerella graminicola LysM effector-mediated stealth pathogenesis subverts recognition through both CERK1 and CEBiP homologues in wheat. Mol. Plant Microbe Interact. 27, 236-243. doi: 10.1094/MPMI-07-13-0201-R

Lefebvre, B., Klaus-Heisen, D., Pietraszewska-Bogiel, A., Herve, C., Camut, S., Auriac, M.-C., et al. (2012). Role of N-glycosylation sites and CXC motifs in trafficking of Medicago truncatula nod factor perception protein to plasma membrane. J. Biol. Chem. 287, 10812-10823. doi: 10.1074/jbc.M111.281634

Leo, J. C., Oberhettinger, P., Chaubey, M., Schütz, M., Kühner, D., Bertsche, U., et al. (2015). The intimin periplasmic domain mediates dimerisation and binding to peptidoglycan. Mol. Microbiol. 95, 80-100. doi: 10.1111/mmi.12840

Leppyanen, I. V., Shakhnazarova, V. Y., Shtark, O. Y., Vishnevskaya, N. A., Tikhonovich, I. A., and Dolgikh, E. A. (2017). Receptor-like kinase LYK9 in Pisum sativum L. Is the CERK1-like receptor that controls both plant immunity and AM symbiosis development. Int. J. Mol. Sci. 19:e8. doi: 10.3390/ ijms 19010008

Lerouge, P., Roche, P., Faucher, C., Maillet, F., Truchet, G., Prome, J. C., et al. (1990). Symbiotic host-specificity of Rhizobium meliloti is determined by a sulphated and acylated glucosamine oligosaccharide signal. Nature 344, 781-784. doi: 10.1038/344781a0

Letunic, I., and Bork, P. (2016). Interactive tree of life (iTOL) v3: an online tool for the display and annotation of phylogenetic and other trees. Nucleic Acids Res. 44, W242-W245. doi: 10.1093/nar/gkw290

Liang, Y., Cao, Y., Tanaka, K., Thibivilliers, S., Wan, J., Choi, J., et al. (2013). Nonlegumes respond to rhizobial nod factors by suppressing the innate immune response. Science 341, 1384-1387. doi: 10.1126/science.1242736

Limpens, E., Franken, C., Smit, P., Willemse, J., Bisseling, T., and Geurts, R. (2003). LysM domain receptor kinases regulating rhizobial Nod factor-induced infection. Science 302, 630-633. doi: 10.1126/science.1090074

Liu, B., Li, J. F., Ao, Y., Qu, J. W., Li, Z. Q., Su, J. B., et al. (2012). Lysin motifcontaining proteins LYP4 and LYP6 play dual roles in peptidoglycan and chitin perception in rice innate immunity. Plant Cell 24, 3406-3419. doi: 10.1105/tpc. 112.102475

Liu, T., Liu, Z., Song, C., Hu, Y., Han, Z., She, J., et al. (2012). Chitin-induced dimerization activates a plant immune receptor. Science 336, 1160-1164. doi: $10.1126 /$ science. 1218867 
Liu, S., Wang, J., Han, Z., Gong, X., Zhang, H., and Chai, J. (2016). Molecular mechanism for fungal cell wall recognition by rice chitin receptor OsCEBiP. Structure 24, 1192-1200. doi: 10.1016/j.str.2016.04.014

Lohmann, G. V., Shimoda, Y., Nielsen, M. W., Jørgensen, F. G., Grossmann, C., Sandal, N., et al. (2010). Evolution and regulation of the Lotus japonicus LysM receptor gene family. Mol. Plant Microbe Interact. 23, 510-521. doi: 10.1094/ MPMI-23-4-0510

Macho, A. P., and Zipfel, C. (2015). Targeting of plant pattern recognition receptortriggered immunity by bacterial type-III secretion system effectors. Curr. Opin. Microbiol. 23, 14-22. doi: 10.1016/j.mib.2014.10.009

Madsen, E. B., Antolín-Llovera, M., Grossmann, C., Ye, J., Vieweg, S., Broghammer, A., et al. (2011). Autophosphorylation is essential for the in vivo function of the Lotus japonicus Nod factor receptor 1 and receptor-mediated signalling in cooperation with Nod factor receptor 5. Plant J. 65, 404-417. doi: 10.1111/j.1365-313X.2010.04431.x

Madsen, E. B., Madsen, L. H., Radutoiu, S., Olbryt, M., Rakwalska, M., Szczyglowski, K., et al. (2003). A receptor kinase gene of the LysM type is involved in legume perception of rhizobial signals. Nature 425, 637-640. doi: 10.1038/nature02045

Maillet, F., Poinsot, V., André, O., Puech-Pagès, V., Haouy, A., Gueunier, M., et al. (2011). Fungal lipochitooligosaccharide symbiotic signals in arbuscular mycorrhiza. Nature 469, 58-63. doi: 10.1038/nature09622

Malkov, N., Fliegmann, J., Rosenberg, C., Gasciolli, V., Timmers, A. C., Nurisso, A., et al. (2016). Molecular basis of lipo-chitooligosaccharide recognition by the lysin motif receptor-like kinase LYR3 in legumes. Biochem. J. 473, 1369-1378. doi: 10.1042/BCJ20160073

Marshall, R., Kombrink, A., Motteram, J., Loza-Reyes, E., Lucas, J., HammondKosack, K. E., et al. (2011). Analysis of two in planta expressed LysM effector homologs from the fungus Mycosphaerella graminicola reveals novel functional properties and varying contributions to virulence on wheat. Plant Physiol. 156, 756-769. doi: 10.1104/pp.111.176347

Maxwell, K. L., Fatehi Hassanabad, M., Chang, T., Paul, V. D., Pirani, N., Bona, D., et al. (2013). Structural and functional studies of gpX of Escherichia coli phage P2 reveal a widespread role for LysM domains in the baseplates of contractiletailed phages. J. Bacteriol. 195, 5461-5468. doi: 10.1128/JB.00805-13

Mbengue, M., Bourdais, G., Gervasi, F., Beck, M., Zhou, J., Spallek, T., et al. (2016). Clathrin-dependent endocytosis is required for immunity mediated by pattern recognition receptor kinases. Proc. Natl. Acad. Sci. U.S.A. 113, 11034-11039. doi: 10.1073/pnas.1606004113

Mélida, H., Sopeńa-Torres, S., Bacete, L., Garrido-Arandia, M., Jordá, L., López, G., et al. (2018). Non-branched $\beta$-1,3-glucan oligosaccharides trigger immune responses in Arabidopsis. Plant J. 93, 34-49. doi: 10.1111/tpj.13755

Mentlak, T. A., Kombrink, A., Shinya, T., Ryder, L. S., Otomo, I., Saitoh, H., et al. (2012). Effector-mediated suppression of chitin-triggered immunity by Magnaporthe oryzae is necessary for rice blast disease. Plant Cell 24, 322-335. doi: $10.1105 /$ tpc.111.092957

Mesnage, S., Dellarole, M., Baxter, N. J., Rouget, J. B., Dimitrov, J. D., Wang, N., et al. (2014). Molecular basis for bacterial peptidoglycan recognition by LysM domains. Nat. Commun. 5:4269. doi: 10.1038/ncomms5269

Miya, A., Albert, P., Shinya, T., Desaki, Y., Ichimura, K., Shirasu, K., et al. (2007). CERK1, a LysM receptor kinase, is essential for chitin elicitor signaling in Arabidopsis. Proc. Natl. Acad. Sci. U.S.A. 104, 19613-19618. doi: 10.1073/pnas. 0705147104

Miyata, K., Hayafune, M., Kobae, Y., Kaku, H., Nishizawa, Y., Masuda, Y., et al. (2016). Evaluation of the role of the LysM receptor-like kinase, OsNFR5/OsRLK2 for AM symbiosis in rice. Plant Cell Physiol. 57, 2283-2290. doi: $10.1093 /$ pcp/pcw144

Miyata, K., Kozaki, T., Kouzai, Y., Ozawa, K., Ishii, K., Asamizu, E., et al. (2014). The bifunctional plant receptor, OsCERK1, regulates both chitin-triggered immunity and arbuscular mycorrhizal symbiosis in rice. Plant Cell Physiol. 55, 1864-1872. doi: 10.1093/pcp/pcu129

Moling, S., Pietraszewska-Bogiel, A., Postma, M., Fedorova, E., Hink, M. A., Limpens, E., et al. (2014). Nod factor receptors form heteromeric complexes and are essential for intracellular infection in Medicago nodules. Plant Cell 26, 4188-4199. doi: 10.1105/tpc.114.129502

Nakagawa, T., Kaku, H., Shimoda, Y., Sugiyama, A., Shimamura, M., Takanashi, K., et al. (2011). From defense to symbiosis: limited alterations in the kinase domain of LysM receptor-like kinases are crucial for evolution of legumeRhizobium symbiosis. Plant J. 65, 169-180. doi: 10.1111/j.1365-313X.2010. 04411.x

Narusaka, Y., Shinya, T., Narusaka, M., Motoyama, N., Shimada, H., Murakami, K., et al. (2013). Presence of LYM2 dependent but CERK1 independent disease resistance in Arabidopsis. Plant Signal. Behav. 8:e25345. doi: 10.4161/psb.25345

Oh, M. H., Wang, X. F., Kota, U., Goshe, M. B., Clouse, S. D., and Huber, S. C. (2009). Tyrosine phosphorylation of the BRI1 receptor kinase emerges as a component of brassinosteroid signaling in Arabidopsis. Proc. Natl. Acad. Sci. U.S.A. 106, 658-663. doi: 10.1073/pnas.0810249106

Okada, M., Matsumura, M., Ito, Y., and Shibuya, N. (2002). High-affinity binding proteins for $\mathrm{N}$-acetylchitooligosaccharide elicitor in the plasma membranes from wheat, barley and carrot cells: conserved presence and correlation with the responsiveness to the elicitor. Plant Cell Physiol. 43, 505-512. doi: 10.1093/ pcp/pcf060

Okazaki, S., Kaneko, T., Sato, S., and Saeki, K. (2013). Hijacking of leguminous nodulation signaling by the rhizobial type III secretion system. Proc. Natl. Acad. Sci. U.S.A. 110, 17131-17136. doi: 10.1073/pnas. 1302360110

Olah, B., Briere, C., Becard, G., Denarie, J., and Gough, C. (2005). Nod factors and a diffusible factor from arbuscular mycorrhizal fungi stimulate lateral root formation in Medicago truncatula via the DMI1/DMI2 signalling pathway. Plant J. 44, 195-207. doi: 10.1111/j.1365-313X.2005.02522.x

Oldroyd, G. E., Mitra, R. M., Wais, R. J., and Long, S. R. (2001). Evidence for structurally specific negative feedback in the Nod factor signal transduction pathway. Plant J. 28, 191-199. doi: 10.1046/j.1365-313X.2001.01149.x

Op den Camp, R., Streng, A., De Mita, S., Cao, Q., Polone, E., Liu, W., et al. (2011). LysM-type mycorrhizal receptor recruited for rhizobium symbiosis in nonlegume Parasponia. Science 331, 909-912. doi: 10.1126/science.1198181

Paparella, C., Savatin, D. V., Marti, L., De Lorenzo, G., and Ferrari, S. (2014). The Arabidopsis LYSIN MOTIF-CONTAINING RECEPTOR-LIKE KINASE3 regulates the cross talk between immunity and abscisic acid responses. Plant Physiol. 165, 262-276. doi: 10.1104/pp.113.233759

Petutschnig, E. K., Jones, A. M., Serazetdinova, L., Lipka, U., and Lipka, V. (2010). The lysin motif receptor-like kinase (LysM-RLK) CERK1 is a major chitin-binding protein in Arabidopsis thaliana and subject to chitin-induced phosphorylation. J. Biol. Chem. 285, 28902-28911. doi: 10.1074/jbc.M110. 116657

Pietraszewska-Bogiel, A., Lefebvre, B., Koini, M. A., Klaus-Heisen, D., Takken, F. L. W., Geurts, R., et al. (2013). Interaction of Medicago truncatula lysin motif receptor-like kinases, NFP and LYK3, produced in Nicotiana benthamiana induces defence- like responses. PLoS One 8:e65055. doi: 10.1371/journal.pone. 0065055

Radutoiu, S., Madsen, L. H., Madsen, E. B., Felle, H. H., Umehara, Y., Gronlund, M., et al. (2003). Plant recognition of symbiotic bacteria requires two LysM receptor-like kinases. Nature 425, 585-592. doi: 10.1038/nature02039

Radutoiu, S., Madsen, L. H., Madsen, E. B., Jurkiewicz, A., Fukai, E., Quistgaard, E. M., et al. (2007). LysM domains mediate lipochitin-oligosaccharide recognition and $\mathrm{Nfr}$ genes extend the symbiotic host range. EMBO J. 26, 3923-3935. doi: 10.1038/sj.emboj.7601826

Rasmussen, S. R., Füchtbauer, W., Novero, M., Volpe, V., Malkov, N., Genre, A., et al. (2016). Intraradical colonization by arbuscular mycorrhizal fungi triggers induction of a lipochitooligosaccharide receptor. Sci. Rep. 6:29733. doi: 10.1038/ srep29733

Rey, T., Chatterjee, A., Buttay, M., Toulotte, J., and Schornack, S. (2015). Medicago truncatula symbiosis mutants affected in the interaction with a biotrophic root pathogen. New Phytol. 206, 497-500. doi: 10.1111/nph.13233

Rey, T., Nars, A., Bonhomme, M., Bottin, A., Huguet, S., Balzergue, S., et al. (2013). NFP, a LysM protein controlling Nod factor perception, also intervenes in Medicago truncatula resistance to pathogens. New Phytol. 198, 875-886. doi: 10.1111/nph.12198

Rose, C. M., Venkateshwaran, M., Volkening, J. D., Grimsrud, P. A., Maeda, J., Bailey, D. J., et al. (2012). Rapid phosphoproteomic and transcriptomic changes in the rhizobia-legume symbiosis. Mol. Cell. Proteomics 11, 724-744. doi: 10. 1074/mcp.M112.019208

Sánchez-Vallet, A., Mesters, J. R., and Thomma, B. P. (2015). The battle for chitin recognition in plant-microbe interactions. FEMS Microbiol. Rev. 39, 171-183. doi: 10.1093/femsre/fuu003 
Sánchez-Vallet, A., Saleem-Batcha, R., Kombrink, A., Hansen, G., Valkenburg, D. J., Thomma, B. P., et al. (2013). Fungal effector Ecp6 outcompetes host immune receptor for chitin binding through intrachain LysM dimerization. eLife 2:e00790. doi: 10.7554/eLife.00790

Schwessinger, B., and Ronald, P. C. (2012). Plant innate immunity: perception of conserved microbial signatures. Annu. Rev. Plant Biol. 63, 451-482. doi: 10.1146/annurev-arplant-042811-105518

Schwessinger, B., Roux, M., Kadota, Y., Ntoukakis, V., Sklenar, J., Jones, A., et al. (2011). Phosphorylation-dependent differential regulation of plant growth, cell death, and innate immunity by the regulatory receptor-like kinase BAK1. PLoS Genet. 7:e1002046. doi: 10.1371/journal.pgen.1002046

Shibuya, N., Ebisu, N., Kamada, Y., Kaku, H., Cohn, J., and Ito, Y. (1996). Localization and binding characteristics of a high-affinity binding site for $\mathrm{N}$-acetylchitooligosaccharide elicitor in the plasma membrane from suspension-cultured rice cells suggest a role as a receptor for the elicitor signal at the cell surface. Plant Cell Physiol. 37, 894-898. doi: 10.1093/oxfordjournals. pcp.a029030

Shibuya, N., Kaku, H., Kuchitsu, K., and Maliarik, M. J. (1993). Identification of a novel high-affinity binding site for $\mathrm{N}$-acetylchitooligosaccharide elicitor in the membrane fraction from suspension-cultured rice cells. FEBS Lett. 329, 75-78. doi: 10.1016/0014-5793(93)80197-3

Shimizu, T., Nakano, T., Takamizawa, D., Desaki, Y., Ishii-Minami, N., Nishizawa, Y., et al. (2010). Two LysM receptor molecules, CEBiP and OsCERK1, cooperatively regulate chitin elicitor signaling in rice. Plant J. 64, 204-214. doi: 10.1111/j.1365-313X.2010.04324.X

Shinya, T., Motoyama, N., Ikeda, A., Wada, M., Kamiya, K., Hayafune, M., et al. (2012). Functional characterization of CEBiP and CERK1 homologs in Arabidopsis and rice reveals the presence of different chitin receptor systems in plants. Plant Cell Physiol. 53, 1696-1706. doi: 10.1093/pcp/pcs113

Shinya, T., Osada, T., Desaki, Y., Hatamoto, M., Yamanaka, Y., Hirano, H., et al. (2010). Characterization of receptor proteins using affinity cross-linking with biotinylated ligands. Plant Cell Physiol. 51, 262-270. doi: 10.1093/pcp/pcp185

Shiu, S. H., and Bleecker, A. B. (2003). Expansion of the receptor-like kinase/Pelle gene family and receptor-like proteins in Arabidopsis. Plant Physiol. 132, 530-543. doi: 10.1104/pp.103.021964

Smit, P., Limpens, E., Geurts, R., Fedorova, E., Dolgikh, E., Gough, C., et al. (2007). Medicago LYK3, an entry receptor in rhizobial nodulation factor signaling. Plant Physiol. 145, 183-191. doi: 10.1104/pp.107.100495

Staehelin, C., Granado, J., Muller, J., Wiemken, A., Mellor, R. B., Felix, G., et al. (1994). Perception of Rhizobium nodulation factors by tomato cells and inactivation by root chitinases. Proc. Natl. Acad. Sci. U.S.A. 91, 2196-2200. doi: 10.1073/pnas.91.6.2196

Sulima, A. S., Zhukov, V. A., Afonin, A. A., Zhernakov, A. I., Tikhonovich, I. A., and Lutova, L. A. (2017). Selection signatures in the first exon of paralogous receptor kinase genes from the. Front. Plant Sci. 8:1957. doi: 10.3389/fpls.2017.01957

Sun, J., Miller, J. B., Granqvist, E., Wiley-Kalil, A., Gobbato, E., Maillet, F., et al. (2015). Activation of symbiosis signaling by arbuscular mycorrhizal fungi in legumes and rice. Plant Cell 27, 823-838. doi: 10.1105/tpc.114.131326

Svistoonoff, S., Hocher, V., and Gherbi, H. (2014). Actinorhizal root nodule symbioses: what is signalling telling on the origins of nodulation? Curr. Opin. Plant Biol. 20, 11-18. doi: 10.1016/j.pbi.2014.03.001

Takahara, H., Hacquard, S., Kombrink, A., Hughes, H. B., Halder, V., Robin, G. P., et al. (2016). Colletotrichum higginsianum extracellular LysM proteins play dual roles in appressorial function and suppression of chitin-triggered plant immunity. New Phytol. 211, 1323-1337. doi: 10.1111/nph.13994

Tanaka, S., Ichikawa, A., Yamada, K., Tsuji, G., Nishiuchi, T., Mori, M., et al. (2010). HvCEBiP, a gene homologous to rice chitin receptor CEBiP, contributes to basal resistance of barley to Magnaporthe oryzae. BMC Plant Biol. 10:288. doi: 10.1186/1471-2229-10-288

van Velzen, R., Holmer, R., Bu, F., Rutten, L., van Zeijl, A., Liu, W., et al. (2018). Comparative genomics of the nonlegume. Proc. Natl. Acad. Sci. U.S.A. 115, E4700-E4709. doi: 10.1073/pnas.1721395115
Wais, R. J., Galera, C., Oldroyd, G., Catoira, R., Penmetsa, R. V., Cook, D., et al. (2000). Genetic analysis of calcium spiking responses in nodulation mutants of Medicago truncatula. Proc. Natl. Acad. Sci. U.S.A. 97, 13407-13412. doi: 10.1073/pnas.230439797

Wan, J., Tanaka, K., Zhang, X., Son, G., Brechenmacher, L., Tran, H., et al. (2012). LYK4, a lysin motif receptor-like kinase, is important for chitin signaling and plant innate immunity in Arabidopsis. Plant Physiol. 160, 396-406. doi: 10. 1104/pp.112.201699

Wan, J., Zhang, X. C., Neece, D., Ramonell, K. M., Clough, S., Kim, S. Y., et al. (2008). A LysM receptor-like kinase plays a critical role in chitin signaling and fungal resistance in Arabidopsis. Plant Cell 20, 471-481. doi: 10.1105/tpc.107. 056754

Wang, W., Xie, Z. P., and Staehelin, C. (2014). Functional analysis of chimeric lysin motif domain receptors mediating Nod factor-induced defense signaling in Arabidopsis thaliana and chitin-induced nodulation signaling in Lotus japonicus. Plant J. 78, 56-69. doi: 10.1111/tpj.12450

Willmann, R., Lajunen, H. M., Erbs, G., Newman, M.-A., Kolb, D., Tsuda, K., et al. (2011). Arabidopsis lysin-motif proteins LYM1 LYM3 CERK1 mediate bacterial peptidoglycan sensing and immunity to bacterial infection. Proc. Natl. Acad. Sci. U.S.A. 108, 19824-19829. doi: 10.1073/pnas.1112862108

Wong, J., Alsarraf, H., Kaspersen, J. D., Pedersen, J. S., Stougaard, J., Thirup, S., et al. (2014). Cooperative binding of LysM domains determines the carbohydrate affinity of a bacterial endopeptidase protein. FEBS J. 281, 1196-1208. doi: 10.1111/febs.12698

Yamaguchi, Y., Huffaker, A., Bryan, A. C., Tax, F. E., and Ryan, C. A. (2010). PEPR2 is a second receptor for the Pep1 and Pep2 peptides and contributes to defense responses in Arabidopsis. Plant Cell 22, 508-522. doi: 10.1105/tpc.109.068874

Yamaguchi, Y., Pearce, G., and Ryan, C. A. (2006). The cell surface leucine-rich repeat receptor for AtPep1, an endogenous peptide elicitor in Arabidopsis, is functional in transgenic tobacco cells. Proc. Natl. Acad. Sci. U.S.A. 103, 10104-10109. doi: 10.1073/pnas.0603729103

Yang, S., Tang, F., Gao, M., Krishnan, H. B., and Zhu, H. (2010). R gene-controlled host specificity in the legume-rhizobia symbiosis. Proc. Natl. Acad. Sci. U.S.A. 107, 18735-18740. doi: 10.1073/pnas.1011957107

Ye, Y. Y., Ding, Y. F., Jiang, Q., Wang, F. J., Sun, J. W., and Zhu, C. (2017). The role of receptor-like protein kinases (RLKs) in abiotic stress response in plants. Plant Cell Rep. 36, 235-242. doi: 10.1007/s00299-016-2084-x

Zeng, L., Velásquez, A. C., Munkvold, K. R., Zhang, J., and Martin, G. B. (2012). A tomato LysM receptor-like kinase promotes immunity and its kinase activity is inhibited by AvrPtoB. Plant J. 69, 92-103. doi: 10.1111/j.1365-313X.2011. 04773.x

Zhang, X., Dong, W., Sun, J., Feng, F., Deng, Y., He, Z., et al. (2015). The receptor kinase CERK1 has dual functions in symbiosis and immunity signalling. Plant J. 81, 258-267. doi: 10.1111/tpj.12723

Zhang, X. C., Cannon, S. B., and Stacey, G. (2009). Evolutionary genomics of LysM genes in land plants. BMC Evol. Biol. 9:183. doi: 10.1186/1471-2148-9-183

Zipfel, C., Kunze, G., Chinchilla, D., Caniard, A., Jones, J. D., Boller, T., et al. (2006). Perception of the bacterial PAMP EF-Tu by the receptor EFR restricts Agrobacterium-mediated transformation. Cell 125, 749-760. doi: 10.1016/j.cell. 2006.03.037

Conflict of Interest Statement: The authors declare that the research was conducted in the absence of any commercial or financial relationships that could be construed as a potential conflict of interest.

Copyright (c) 2018 Buendia, Girardin, Wang, Cottret and Lefebvre. This is an openaccess article distributed under the terms of the Creative Commons Attribution License (CC BY). The use, distribution or reproduction in other forums is permitted, provided the original author(s) and the copyright owner(s) are credited and that the original publication in this journal is cited, in accordance with accepted academic practice. No use, distribution or reproduction is permitted which does not comply with these terms. 